\title{
Stability in constrained temperature-rate-dependent thermoelasticity
}

\author{
A. M. Alharbi* and N. H. Scott ${ }^{\dagger}$ \\ 5 August 2017
}

\begin{abstract}
In an anisotropic temperature-rate-dependent thermoelastic material four plane harmonic waves may propagate in any direction, all dispersive and attenuated, and all stable in the sense that their amplitudes remain bounded in the direction of travel. In this paper, the material is additionally assumed to suffer an internal constraint of the deformation-temperature type, i.e. the temperature is a prescribed function of the deformation gradient. In this constrained thermoelastic material four waves continue to propagate but instabilities are now found. Constrained temperaturerate-dependent thermoelasticity is then combined with generalized thermoelasticity in which the rate of change of heat flux also appears in the heat conduction equation. Four waves again propagate but instabilities are found as before. Anisotropic and isotropic materials are both considered.
\end{abstract}

\section{Keywords}

Thermoelasticity, generalized thermoelasticity, deformation-temperature constraint, deformation-entropy constraint, second sound, stability.

\section{Introduction}

Truesdell and Noll [1] placed the theory of purely mechanical constraints on a firm theoretical basis by postulating that the stress is constitutively determined only to within a reaction stress that does no work in any motion satisfying the constraint. By contrast, there is more than one way of treating the general theory of the thermomechanical constraint viewed as a restriction on the allowable values of the deformation gradient and temperature. In the thermomechanical case, Green et al. [2] supposed that the stress, entropy and heat flux led to zero entropy production in any process satisfying the thermomechanical constraint.

*Department of Mathematics, Faculty of Science, Taif University, Saudi Arabia. Email: alharbi.a.m@hotmail.com

†Corresponding author: School of Mathematics, University of East Anglia, Norwich NR4 7TJ, UK. Email: n.scott@uea.ac.uk 
Casey and Krishanaswamy [3] developed an alternative type of theory for deformationtemperature constraints. They obtained expressions for the stress and entropy in the constrained material by considering a related family of unconstrained thermoelastic materials, which itself was obtained by extending the domain of definition of the Helmholtz free energy, in a differentiable manner, away from the constraint manifold.

A linearized theory of plane wave propagation in thermoelastic materials was developed by Chadwick $[4,5]$, and he established that four wave modes are possible in each direction. Scott [6] proved that each of these modes is stable under quite mild assumptions. We define a stable wave as a wave whose amplitude remains bounded in the direction of propagation. For an isothermal or isentropic elastic material we recall that three stable waves may propagate in each direction and that only two stable waves propagate if a purely mechanical constraint operates, see Chadwick et al. [7]. Thus the presence of a purely mechanical constraint removes one mode of propagation but maintains the stability of the system.

A natural choice of thermomechanical constraint might be one that connects deformation with temperature. This would appear to be well motivated, as materials as diverse as vulcanized rubbers and water are considered to be incompressible at a fixed temperature, though the material is permitted to expand if the fixed temperature is increased. For a fully anisotropic material suffering an arbitrary deformation-temperature constraint, Chadwick and Scott [8] showed that of the four waves that propagate at least one is unstable, in contrast with the purely mechanical constraint. Scott [9] and Leslie and Scott $[10,11,12]$ have developed these constraint theories further but in all circumstances have encountered instabilities.

Green and Lindsay [13] formulated a theory of thermoelasticity with second sound based on an entropy production inequality proposed by Green and Laws [14]. A noteworthy feature of the Green and Lindsay theory is that it retains the classical Fourier law if the material has a centre of symmetry at each point. In the present work we follow Green and Lindsay theory, which we refer to as temperature-rate-dependent thermoelasticity theory.

The classical Fourier heat conduction law for anisotropic materials is

$$
q_{i}=-k_{i j} \theta_{, j}
$$

in which $q_{i}(\mathbf{x}, t)$ are the components of the heat flux vector $\mathbf{q}$ and $\theta(\mathbf{x}, t)$ is the temperature increment, both of which are functions of particle position $\mathbf{x}$ and time $t$. The notation ()$_{, j}$ denotes the spatial derivative $\partial() / \partial x_{j}$ and the summation convention applies so that twiceoccurring suffices are summed over. The quantities $k_{i j}$ are the components of the thermal conductivity tensor $\mathbf{k}$. The symmetry of $\mathbf{k}$, i.e. $k_{i j}=k_{j i}$, is part of the infrastructure of temperature-rate-dependent thermoelasticity though not of classical thermoelasticity.

In the generalized thermoelasticity theory of Lord and Shulman [15] the heat flux vector components $q_{i}(\mathbf{x}, t)$ satisfy

$$
\left(1+\tau \frac{\partial}{\partial t}\right) q_{i}=-k_{i j} \theta_{, j}
$$

in place of equation (1) in which the constant $\tau>0$ is a relaxation time. It is clear that when $\tau=0$, equation (2) reduces to equation (1) of the classical case. 
Scott [16] has demonstrated the linear stability of the Lord and Shulman [15] model of generalized thermoelasticity, i.e. the combination of the modified Fourier law (2) with classical thermoelasticity.

We pursue here a method of combining temperature-rate-dependent thermoelasticity [13] and generalized thermoelasticity [15] due to Ignaczak [17] in the isotropic case.

Chandrasekharaiah [18] presented a broad review of the literature concerned with generalized thermoelasticity theories, including a brief account of the theory of heat conduction with second sound. Straughan [19] has given an excellent account of many theories involving hyperbolic heat conduction; i.e. the propagation of heat waves.

The present authors have given detailed accounts of the stability theory of temperaturerate-dependent thermoelasticity for isotropic materials, see Alharbi and Scott [20], and for anisotropic materials, see Alharbi and Scott [21]. In all cases they found all linear waves to be stable. Alharbi and Scott [20, 21] also considered generalized temperature-ratedependent thermoelasticity but found instabilities.

In this paper we derive the linearized field equations for temperature-rate-dependent thermoelasticity when the material suffers a deformation-temperature constraint. Solutions of the field equation are sought in the form of plane harmonic waves and the secular equation is found for the squared wave speed $w(\omega)$, considered as a function of the frequency $\omega$. In all cases there are four branches of $w(\omega)$ in the complex $w$-plane as $\omega$ varies. Low- and high-frequency expansions are performed for each branch and the stability or instability of the branch established, considering both anisotropic and isotropic thermoelastic materials. We then combine the theory of temperature-rate-dependent thermoelasticity with the generalized thermoelasticity theory of Lord and Shulman [15] and once again assume the material suffers a deformation-temperature constraint. As before, we find that there are four branches $w(\omega)$ and determine their stability or instability in both the anisotropic and isotropic cases.

The paper is organized as follows. In Section 2 the field equations for various models of thermoelasticity are derived: unconstrained and constrained classical thermoelasticity, unconstrained and constrained temperature-rate-dependent thermoelasticity, and constrained generalized temperature-rate-dependent thermoelasticity. In Section 3 we consider the theory of constrained anisotropic temperature-rate-dependent thermoelasticity and in Section 4 we consider the isotropic case. In Section 5 we consider the theory of constrained anisotropic generalized temperature-rate-dependent thermoelasticity and in Section 6 we consider the isotropic case. The final section contains a discussion of our results. 


\section{Field equations for various models of thermoelastic- ity}

We consider a thermoelastic body which possesses a spatially uniform, time-independent, stress-free equilibrium state free of heat flux. For a body with such an equilibrium state the equations of momentum and energy balance in the absence of body force and heat supply, linearized about this equilibrium state, are

$$
\sigma_{i j, j}=\rho \ddot{u}_{i}, \quad-q_{i, i}=\rho T \dot{\phi},
$$

respectively, see [5], where $\sigma_{i j}$ and $q_{i}$ are the components of the Cauchy stress tensor and the heat flux vector, respectively. The particle displacement vector $\mathbf{u}(\mathbf{x}, t)$, and the entropy increment $\phi(\mathbf{x}, t)$ per unit mass are functions of particle position $\mathbf{x}$ and time $t$. The superposed dot denotes the time partial derivative. The constant equilibrium values of the density and absolute temperature are denoted by $\rho$ and $T$, respectively.

We recapitulate various models of unconstrained and constrained thermoelastic materials already discussed in the literature and introduce the constrained temperature-ratedependent models we propose to discuss.

\subsection{Unconstrained classical thermoelasticity}

In classical thermoelasticity the Helmholtz free energy $\psi\left(u_{i, j}, \theta\right)$, defined by

$$
\psi\left(u_{i, j}, \theta\right)=\frac{1}{2} \rho^{-1} \tilde{c}_{i j k l} u_{i, j} u_{k, l}-\rho^{-1} \beta_{i j} u_{i, j} \theta-\frac{c}{2 T} \theta^{2},
$$

acts as a potential for the stress and entropy:

$$
\sigma_{i j}=\rho \frac{\partial \psi}{\partial u_{i, j}}, \quad \phi=-\frac{\partial \psi}{\partial \theta},
$$

see, for example, [9, equation (2.6)]. In equation (4), $\tilde{c}_{i j k l}$ are the isothermal elasticity tensor components, $\beta_{i j}$ are the temperature coefficients of stress and $c$ is the specific heat at constant deformation, all measured in the equilibrium state. The temperature excess above the equilibrium temperature $T$ is denoted by $\theta(\mathbf{x}, t)$.

From equations (4), (5) and (1), expressions for the stress, entropy increment and heat flux in the linear theory of classical thermoelasticity are given respectively by

$$
\begin{aligned}
\sigma_{i j} & =\tilde{c}_{i j k l} u_{k, l}-\beta_{i j} \theta, \\
\phi & =\rho^{-1} \beta_{i j} u_{i, j}+T^{-1} c \theta, \\
q_{i} & =-k_{i j} \theta, j .
\end{aligned}
$$

We insert the constitutive equations (6) into the balance equations (3) to obtain

$$
\begin{aligned}
& \tilde{c}_{i j k l} u_{k, j l}-\beta_{i j} \theta_{, j}=\rho \ddot{u}_{i}, \\
& k_{i j} \theta_{, i j}-T \beta_{i j} \dot{u}_{i, j}=\rho c \dot{\theta},
\end{aligned}
$$

the field equations of classical linear thermoelasticity theory for anisotropic materials, see $[5$, equations $(19)]$ or $[9$, equations $(2.8 \mathrm{a})-(2.8 \mathrm{~b})]$. These equations provide four constantcoefficient, linear partial differential equations for the four unknown functions $u_{i}$ and $\theta$. 


\subsection{Constrained classical thermoelasticity}

The deformation-temperature constraint usually considered in the literature takes the linearized form

$$
\tilde{N}_{p q} u_{p, q}-\alpha \theta=0
$$

in which $\tilde{\mathbf{N}}$ is the symmetric constraint tensor and $\alpha$ is a coefficient of thermal expansion. With this constraint, the temperature $\theta$ is effectively a prescribed function of the displacement gradient $u_{p, q}$. To account for the constraint, a quantity

$$
\rho^{-1} \tilde{\eta}\left(\tilde{N}_{p q} u_{p, q}-\alpha \theta\right)
$$

see [8], where $\tilde{\eta}$ is a Lagrange multiplier, is added to $\psi$ in equation (4) and then the differentiations in equation (5) give the additional constraint stress $\tilde{\eta} \tilde{\mathbf{N}}$ and the additional constraint entropy $\rho^{-1} \alpha \tilde{\eta}$, so that equations (6) are replaced by

$$
\begin{aligned}
\sigma_{i j} & =\tilde{c}_{i j k l} u_{k, l}-\beta_{i j} \theta+\tilde{N}_{i j} \tilde{\eta}, \\
\phi & =\rho^{-1} \beta_{i j} u_{i, j}+T^{-1} c \theta+\rho^{-1} \alpha \tilde{\eta}, \\
q_{i} & =-k_{i j} \theta_{, j}
\end{aligned}
$$

leading to the five field equations

$$
\begin{aligned}
\tilde{c}_{i j k l} u_{k, j l}-\beta_{i j} \theta_{, j}+\tilde{N}_{i j} \tilde{\eta}_{, j} & =\rho \ddot{u}_{i}, \\
k_{i j} \theta_{, i j}-T \beta_{i j} \dot{u}_{i, j}-T \alpha \dot{\tilde{\eta}} & =\rho c \dot{\theta}, \\
\tilde{N}_{p q} u_{p, q}-\alpha \theta & =0,
\end{aligned}
$$

of constrained classical thermoelasticity, see Chadwick and Scott [8, equations (2.15) and (2.16)], in place of the four field equations (7) of unconstrained classical thermoelasticity. Equation $(10)_{3}$ is simply the constraint equation (8) repeated. These equations provide a set of five equations for the five unknowns $u_{i}, \theta$ and $\tilde{\eta}$.

\subsection{Unconstrained temperature-rate-dependent thermoelasticity}

The stress, entropy increment and heat flux system of equations is given by Chandrasekharaiah $[18$, equations $(5.11)-(5.13)]$

$$
\begin{aligned}
\sigma_{i j} & =\tilde{c}_{i j k l} u_{k, l}-\beta_{i j}\left(1+\alpha_{1} \frac{\partial}{\partial t}\right) \theta, \\
\phi & =\rho^{-1} \beta_{i j} u_{i, j}+T^{-1} c\left(1+\alpha_{0} \frac{\partial}{\partial t}\right) \theta, \\
q_{i} & =-k_{i j} \theta_{, j},
\end{aligned}
$$

for materials with a centre of symmetry at each point. The new material constants $\alpha_{1}$ and $\alpha_{0}$ are relaxation times. Green and Lindsay [13] show that

$$
\alpha_{1} \geq \alpha_{0} \geq 0
$$


is a requirement of the second law of thermodynamics.

We insert equation (11) into equation (3) to get the field equations of temperature-ratedependent thermoelasticity in the form

$$
\begin{aligned}
\tilde{c}_{i j k l} u_{k, j l}-\beta_{i j}\left(\theta+\alpha_{1} \dot{\theta}\right)_{, j} & =\rho \ddot{u}_{i}, \\
k_{i j} \theta_{, i j}-T \beta_{i j} \dot{u}_{i, j} & =\rho c\left(\dot{\theta}+\alpha_{0} \ddot{\theta}\right) .
\end{aligned}
$$

These equations form a complete system of field equations for linear temperature-ratedependent thermoelasticity for a homogeneous and anisotropic material and provide four constant-coefficient, linear partial differential equations for the four unknown functions $u_{i}$ and $\theta$, see [18, equations (5.17)-(5.18)]. By setting $\alpha_{1}=\alpha_{0}=0$, we recover the field equations of linear classical thermoelasticity theory for homogeneous and anisotropic solids, see $[9$, equations $(2.8 \mathrm{a})-(2.8 \mathrm{~b})]$.

\subsection{Constrained temperature-rate-dependent thermoelasticity}

In their theory of temperature rate dependent thermoelasticity Green and Lindsay [13] introduce a second temperature function $\theta_{1}(\theta, \dot{\theta})$ which plays an important role in entropy production (and is denoted by $\phi$ in [13]). They show that equation (5) must be replaced by

$$
\sigma_{i j}=\rho \frac{\partial \psi}{\partial u_{i, j}}, \quad \phi=-\frac{\partial \psi}{\partial \dot{\theta}} / \frac{\partial \theta_{1}}{\partial \dot{\theta}},
$$

see $\left[13\right.$, equations $(3.15)_{1}$ and (3.6)]. We shall replace the constraint equation (8) of the classical theory by

$$
\tilde{N}_{p q} u_{p, q}-\alpha \theta_{1}=0,
$$

in which the usual temperature $\theta$ has been replaced by the second temperature $\theta_{1}$. To account for the new constraint, a quantity $\rho^{-1} \tilde{\eta}\left(\tilde{N}_{p q} u_{p, q}-\alpha \theta_{1}\right)$, where $\tilde{\eta}$ is again a Lagrange multiplier, is added to $\psi$ and then the differentiations (14) give the additional constraint stress $\tilde{\eta} \tilde{\mathbf{N}}$ and the additional constraint entropy $\rho^{-1} \alpha \tilde{\eta}$ exactly as occurred in equation $(9)_{1,2}$.

It follows that the other field equations of the anisotropic case $(10)_{1,2}$ also hold in the present case:

$$
\begin{aligned}
\tilde{c}_{i j k l} u_{k, j l}-\beta_{i j}\left(\theta_{, j}+\alpha_{1} \dot{\theta}_{, j}\right)+\tilde{N}_{i j} \tilde{\eta}_{, j} & =\rho \ddot{u}_{i}, \\
k_{i j} \theta_{, i j}-T \beta_{p q} \dot{u}_{p, q}-T \alpha \dot{\tilde{\eta}} & =\rho c\left(\dot{\theta}+\alpha_{0} \ddot{\theta}\right), \\
\tilde{N}_{p q} u_{p, q}-\alpha\left(\theta+\alpha_{0} \dot{\theta}\right) & =0,
\end{aligned}
$$

the third equation here simply being the constraint equation (15) written in terms of the linearized form [13, equation (4.4)] of $\theta_{1}$ :

$$
\theta_{1}=\theta+\alpha_{0} \dot{\theta}
$$

It is with the field equations (16), which are new to the literature, that we shall be concerned in Sections 3 and 4. 


\subsection{Constrained generalized temperature-rate-dependent thermoe- lasticity}

We combine the temperature-rate-dependent thermoelasticity theory of Green and Lindsay [13] with the generalized thermoelasticity theory of Lord and Shulman [15] by following the method of Ignaczak [17] in the isotropic case To do this we simply replace Fourier's law of heat conduction (1) by Lord and Shulman's equation (2) which has the effect of replacing the field equations (16) by

$$
\begin{aligned}
\tilde{c}_{i j k l} u_{k, j l}-\beta_{i j}\left(\theta, j+\alpha_{1} \dot{\theta}_{, j}\right)+\tilde{N}_{i j} \tilde{\eta}_{, j} & =\rho \ddot{u}_{i}, \\
k_{i j} \theta_{, i j}-T \beta_{p q}\left(1+\tau \frac{\partial}{\partial t}\right) \dot{u}_{p, q}-T \alpha\left(1+\tau \frac{\partial}{\partial t}\right) \dot{\tilde{\eta}} & =\rho c\left(1+\tau \frac{\partial}{\partial t}\right)\left(\dot{\theta}+\alpha_{0} \ddot{\theta}\right), \\
\tilde{N}_{p q} u_{p, q}-\alpha\left(\theta+\alpha_{0} \dot{\theta}\right) & =0 .
\end{aligned}
$$

Equations $(18)_{1,3}$ are exactly the same as equations $(16)_{1,3}$ and equation $(18)_{2}$ differs from equation $(16)_{2}$ only by the introduction of the factors $1+\tau \partial / \partial t$. If $\tau=0$, equations (18) reduce to equations (16), as expected.

We shall be concerned with the field equations (18), which are new to the literature, in Sections 5 and 6. 


\section{Constrained anisotropic temperature-rate-dependent thermoelasticity}

We are concerned with solutions of equations (16) in the form of plane harmonic waves

$$
\left\{u_{i}, \theta, \tilde{\eta}\right\}=\left\{U_{i}, \Theta, \tilde{H}\right\} \exp \{i \omega(s \mathbf{n} \cdot \mathbf{x}-t)\},
$$

where $\omega$ is the angular frequency and $\mathbf{n}$ is the unit wave normal vector in the direction of propagation, both of which are real constants. The amplitudes $\left\{U_{i}, \Theta, \tilde{H}\right\}$ and slowness $s$ are in general complex constants. The wave slowness $s$ is the reciprocal of the (complex) wave speed $v: s=1 / v$. The quantity with which we shall mostly be concerned is the squared wave speed $w=\rho s^{-2}$.

For a wave mode to be linearly stable requires the condition of stability

$$
\operatorname{Im} w(\omega) \leq 0
$$

for $0 \leq \omega<\infty$, see [6, equation (18)] and [21, Section 2.1.1]. So for positive $\omega$, stable branches $w(\omega)$ are those which lie in the lower half of the complex $w$-plane.

Much of the analysis in this section is an extension of that of Alharbi and Scott [21] for unconstrained anisotropic temperature-rate-dependent thermoelasticity.

\subsection{The secular equation}

We look for solutions of equations (16) in the form of the plane harmonic waves (19) by inserting equation (19) into equation (16). Divide the first of the resulting equations by $(i \omega s)^{2} \gamma$ and the second by $i \omega$ to obtain, after rearranging,

$$
\begin{aligned}
\left(\tilde{Q}_{i k}-w \delta_{i k}\right) U_{k}-(i \omega s)^{-1} \gamma^{-1} b_{i}\left(1-i \omega \alpha_{1}\right) \Theta+(i \omega s)^{-1} \gamma^{-1} \tilde{c}_{i} \tilde{H} & =0 \\
(i \omega s) T b_{k} U_{k}+\left[(i \omega s) s k+\rho c\left(1-i \omega \alpha_{0}\right)\right] \Theta+\alpha T \tilde{H} & =0 \\
(i \omega s) \tilde{c}_{k} U_{k}-\alpha\left(1-i \omega \alpha_{0}\right) \Theta & =0 .
\end{aligned}
$$

We have introduced the positive constant $\gamma$, with the physical dimensions of stress, so as to facilitate the non-dimensionalization of our equations. It is usually selected to be a typical component of the isothermal elasticities $\tilde{c}_{i j k l}$.

In addition to the quantity $\gamma$ we have introduced into equations (21) the quantities

$$
\tilde{Q}_{i k}=\gamma^{-1} \tilde{c}_{i j k l} n_{j} n_{l}, \quad w=\rho s^{-2} / \gamma, \quad b_{i}=\beta_{i j} n_{j}, \quad \tilde{c}_{p}=\tilde{N}_{p q} n_{q}, \quad k=k_{i j} n_{i} n_{j}
$$

in which $\tilde{\mathbf{Q}}(\mathbf{n})$ is the dimensionless isothermal acoustic tensor and $w=\rho v^{2} / \gamma$ is the dimensionless squared wave speed. The quantities $\mathbf{b}$ and $k$ are not dimensionless but $\tilde{\mathbf{c}}$ is.

Eliminating $\Theta$ and $\tilde{H}$ from equation $(21)_{1}$ in favour of $\mathbf{U}$ using equations $(21)_{2,3}$ leads, after much reduction, to

$$
\mathrm{MU}=\mathbf{0},
$$


where $\mathbf{M}$ is defined by

$$
\mathbf{M}=\tilde{\mathbf{Q}}-w \mathbf{1}-\frac{1-i \omega \alpha_{1}}{\alpha \gamma\left(1-i \omega \alpha_{0}\right)} \mathbf{b} \otimes \tilde{\mathbf{c}}-\frac{1}{\alpha \gamma} \tilde{\mathbf{c}} \otimes \mathbf{b}-\frac{\rho c}{\alpha \gamma T}\left[1+\frac{i \omega / \omega^{*}}{w\left(1-i \omega \alpha_{0}\right)}\right] \tilde{\mathbf{c}} \otimes \tilde{\mathbf{c}}
$$

in which $\omega^{*}=\gamma c / k$ is a reference frequency and $\otimes$ denotes the dyadic, or tensor, product. Although $\mathbf{M}$ is dimensionless not all the quantities appearing in it are themselves dimensionless. In order to facilitate the full non-dimensionalization of $\mathbf{M}$, we utilize the frequency $\omega^{*}$ in order to define dimensionless forms for the frequency $\omega$ and the relaxation times $\alpha_{0}$ and $\alpha_{1}$, respectively:

$$
\omega^{\prime}=\omega / \omega^{*}, \quad \alpha_{0}^{\prime}=\alpha_{0} \omega^{*}, \quad \alpha_{1}^{\prime}=\alpha_{1} \omega^{*}, \quad c^{\prime}=\frac{\rho c T}{\gamma}, \quad \alpha^{\prime}=\alpha T .
$$

The quantity $c^{\prime}$ is a dimensionless specific heat, the ratio of heat energy to strain energy, and $\alpha^{\prime}$ is the dimensionless thermal expansion coefficient associated with the constraint.

The dimensionless unit vectors $\mathbf{b}^{\prime}$ and $\tilde{\mathbf{c}}^{\prime}$, the dimensionless thermomechanical coupling constant $\varepsilon$ and the dimensionless constraint ratio $\tilde{\sigma}$ are defined, respectively, by

$$
\mathbf{b}^{\prime}=(\mathbf{b} \cdot \mathbf{b})^{-1 / 2} \mathbf{b}, \quad \tilde{\mathbf{c}}^{\prime}=(\tilde{\mathbf{c}} \cdot \tilde{\mathbf{c}})^{-1 / 2} \tilde{\mathbf{c}}, \quad \varepsilon=\frac{T \mathbf{b} \cdot \mathbf{b}}{\rho c \gamma}, \quad \tilde{\sigma}=\frac{\left(c^{\prime}\right)^{1 / 2}(\tilde{\mathbf{c}} \cdot \tilde{\mathbf{c}})^{1 / 2}}{\alpha^{\prime}} .
$$

The magnitude of the quantity $\tilde{\sigma}$ is a measure of the relative importance of mechanical and thermal effects upon the constraint. If $\tilde{\sigma} \rightarrow 0$ (i.e. $\alpha^{\prime} \rightarrow \infty$ ) the constraint is purely mechanical in nature and has no effect on the temperature; if $\tilde{\sigma} \rightarrow \infty$ (i.e. $\alpha^{\prime} \rightarrow 0$ ) the constraint is purely thermal in nature, forcing the material to be isothermal.

We now introduce the quantities defined in equations (25) and (26) into equation (24) in order to obtain an expression for $\mathbf{M}$ in which all quantities appearing are dimensionless:

$$
\mathbf{M}=\tilde{\mathbf{P}}-w \mathbf{1}+\frac{i \omega \tilde{\sigma}}{1-i \omega \alpha_{0}}\left[\varepsilon^{1 / 2}\left(\alpha_{1}-\alpha_{0}\right) \mathbf{b}-\frac{\tilde{\sigma}}{w} \tilde{\mathbf{c}}\right] \otimes \tilde{\mathbf{c}} .
$$

We have dropped the primes for convenience and introduced the dimensionless tensor

$$
\tilde{\mathbf{P}}=\hat{\mathbf{Q}}-\left(\varepsilon^{1 / 2} \mathbf{b}+\tilde{\sigma} \tilde{\mathbf{c}}\right) \otimes\left(\varepsilon^{1 / 2} \mathbf{b}+\tilde{\sigma} \tilde{\mathbf{c}}\right)
$$

in which

$$
\hat{\mathbf{Q}}=\tilde{\mathbf{Q}}+\varepsilon \mathbf{b} \otimes \mathbf{b}
$$

is the dimensionless isentropic acoustic tensor. In passing from equation (24) to equation (27) we have singled out for future convenience the terms in $\mathbf{M}$ independent of $\omega$, namely, $\tilde{\mathbf{P}}-w 1$.

From the usual symmetries $\tilde{c}_{i j k l}=\tilde{c}_{k l i j}$ and the definition (29), we see that both $\hat{\mathbf{Q}}$ and $\tilde{\mathbf{Q}}$ are symmetric and so each has three real eigenvalues. The dimensionless eigenvalues of $\hat{\mathbf{Q}}$ are denoted by $\hat{q}_{i}, i=1,2,3$, and the dimensionless eigenvalues of $\tilde{\mathbf{Q}}$ are denoted by $\tilde{q}_{i}, i=1,2,3$. If these eigenvalues are distinct they possess the interlacing property

$$
0<\tilde{q}_{1}<\hat{q}_{1}<\tilde{q}_{2}<\hat{q}_{2}<\tilde{q}_{3}<\hat{q}_{3}
$$


as was demonstrated in [6]. The inequality $0<\tilde{q}_{1}$ follows from the assumed positive definiteness of $\tilde{\mathbf{Q}}$, see [6]. If some or all of these eigenvalues coincide then some or all of the occurrences of $<$ in inequalities (30), other than the first, may be replaced by $\leq$, see [6] for a discussion of coincident eigenvalues.

Taking the trace of equation (29) gives the following connection between the coupling constant and the eigenvalues of $\hat{\mathbf{Q}}$ and $\tilde{\mathbf{Q}}$ :

$$
\varepsilon=\hat{q}_{1}+\hat{q}_{2}+\hat{q}_{3}-\tilde{q}_{1}-\tilde{q}_{2}-\tilde{q}_{3}
$$

From inequalities (30) we see that $\varepsilon>0$ which may be replaced by the equality $\varepsilon=0$ only if the isothermal and isentropic eigenvalues coincide in pairs, i.e. $\tilde{q}_{i}=\hat{q}_{i}$ for $i=1,2,3$.

We quote the identity

$$
\operatorname{det}(\mathbf{A}+\alpha \mathbf{b} \otimes \mathbf{c})=\operatorname{det} \mathbf{A}+\alpha \mathbf{b} \cdot \mathbf{A}^{\text {adj }} \mathbf{c},
$$

valid for arbitrary $\alpha, \mathbf{b}, \mathbf{c}$ and $\mathbf{A}$, see [9], with the superscript adj denoting the adjugate of a tensor, i.e., in matrix terms, the transposed matrix of cofactors. From equation (23) the secular equation takes the form $\operatorname{det} \mathbf{M}=0$ and so from equations (27) and (32) it becomes

$$
\operatorname{det}(\tilde{\mathbf{P}}-w \mathbf{1})+\frac{i \omega \tilde{\sigma}}{1-i \omega \alpha_{0}}\left[\varepsilon^{1 / 2}\left(\alpha_{1}-\alpha_{0}\right) \mathbf{b}-\frac{\tilde{\sigma}}{w} \tilde{\mathbf{c}}\right] \cdot(\tilde{\mathbf{P}}-w \mathbf{1})^{\operatorname{adj}} \tilde{\mathbf{c}}=0
$$

We specialize to the case where the material is thermally isotropic, though not mechanically isotropic, and the constraint is one of incompressibility at constant temperature, so that

$$
\beta_{i j}=\beta \delta_{i j}, \quad k_{i j}=k \delta_{i j}, \quad \tilde{N}_{i j}=\delta_{i j},
$$

where $\beta$ is the scalar temperature coefficient of stress and $k$ is the scalar thermal conductivity, leading to

$$
\tilde{\mathbf{c}}=\mathbf{b}=\mathbf{n} .
$$

Then equation (33) simplifies to give, on multiplication by $w$,

$$
w \operatorname{det}(w \mathbf{1}-\tilde{\mathbf{P}})-\frac{i \omega \tilde{\sigma}}{1-i \omega \alpha_{0}}\left[w \varepsilon^{1 / 2}\left(\alpha_{1}-\alpha_{0}\right)-\tilde{\sigma}\right] \mathbf{n} \cdot(w \mathbf{1}-\tilde{\mathbf{P}})^{\text {adj }} \mathbf{n}=0
$$

with equation (28) reducing to

$$
\tilde{\mathbf{P}}=\tilde{\mathbf{Q}}+\left[\varepsilon-\left(\varepsilon^{1 / 2}+\tilde{\sigma}\right)^{2}\right] \mathbf{n} \otimes \mathbf{n} .
$$

Equation (35) is the dimensionless secular equation for anisotropic temperature-ratedependent thermoelasticity which is constrained by the deformation-temperature constraint $(16)_{3}$. It is a quartic equation in the dimensionless squared wave speed $w$.

Selecting a coordinate system based on the eigenvectors of $\tilde{\mathbf{P}}$ we may write

$$
\mathbf{n} \cdot(w \mathbf{1}-\tilde{\mathbf{P}})^{\text {adj }} \mathbf{n}=n_{1}^{2}\left(w-\tilde{p}_{2}\right)\left(w-\tilde{p}_{3}\right)+n_{2}^{2}\left(w-\tilde{p}_{1}\right)\left(w-\tilde{p}_{3}\right)+n_{3}^{2}\left(w-\tilde{p}_{1}\right)\left(w-\tilde{p}_{2}\right)
$$


The right-hand side of equation (37) is quadratic in $w$ and has two zeros $\tilde{W}_{1}$ and $\tilde{W}_{2}$ which satisfy

$$
\tilde{p}_{1}<\tilde{W}_{1}<\tilde{p}_{2}<\tilde{W}_{2}<\tilde{p}_{3} .
$$

The secular equation (35) may therefore be written as

$$
\tilde{F}(w)-\frac{i \omega \tilde{\sigma}}{1-i \omega \alpha_{0}}\left[w \varepsilon^{1 / 2}\left(\alpha_{1}-\alpha_{0}\right)-\tilde{\sigma}\right] \tilde{G}(w)=0
$$

in which $\tilde{F}(w)$ and $\tilde{G}(w)$ are defined by

$$
\tilde{F}(w)=w\left(w-\tilde{p}_{1}\right)\left(w-\tilde{p}_{2}\right)\left(w-\tilde{p}_{3}\right), \quad \tilde{G}(w)=\left(w-\tilde{W}_{1}\right)\left(w-\tilde{W}_{2}\right) .
$$

On putting $\alpha_{1}=\alpha_{0}=0$ in equation (39) we recover the secular equation of an anisotropic material which is constrained by a deformation-temperature constraint in classical thermoelasticity, see [9, equation (3.6)].

From the further identity

$$
\mathbf{n} \cdot(w \mathbf{1}-\tilde{\mathbf{P}})^{\text {adj }} \mathbf{n}=\mathbf{n} \cdot(w \mathbf{1}-\tilde{\mathbf{Q}})^{\text {adj }} \mathbf{n},
$$

see [9], we observe that $\tilde{W}_{1}$ and $\tilde{W}_{2}$ satisfy the further inequalities

$$
\tilde{q}_{1}<\tilde{W}_{1}<\tilde{q}_{2}<\tilde{W}_{2}<\tilde{q}_{3}
$$

in addition to equation (38), from which it follows that

$$
\tilde{W}_{1}>0
$$

because of the positive-definiteness assumption $\tilde{q}_{1}>0$.

In the remainder of this section each of the four branches $w(\omega)$ of the secular equation (39) is examined in detail, with low- and high-frequency expansions being performed, and stability or instability proved for the entire frequency range.

\subsection{Low-frequency expansions}

When $\omega=0$, the roots of the secular equation (39) are the zeros of $\tilde{F}(w)$, namely, $\tilde{p}_{i}, i=$ $0,1,2,3$, defining $\tilde{p}_{0} \equiv 0$. When $\omega \rightarrow 0$, the four roots of the secular equation may be obtained as the Taylor expansions

$$
w_{i}(\omega)=\tilde{p}_{i}+i \omega \tilde{\sigma}\left[\tilde{p}_{i} \varepsilon^{\frac{1}{2}}\left(\alpha_{1}-\alpha_{0}\right)-\tilde{\sigma}\right] \frac{\tilde{G}\left(\tilde{p}_{i}\right)}{\tilde{F}^{\prime}\left(\tilde{p}_{i}\right)}, \quad i=0,1,2,3,
$$

neglecting terms $O\left(\omega^{2}\right)$. When $i=0$, so that $\tilde{p}_{i}=\tilde{p}_{0}=0$, equation (44) reduces to

$$
w_{0}(\omega)=-i \omega \tilde{\sigma}^{2} \frac{\tilde{G}\left(\tilde{p}_{0}\right)}{\tilde{F}^{\prime}\left(\tilde{p}_{0}\right)}=i \omega \tilde{\sigma}^{2} \frac{\tilde{W}_{1} \tilde{W}_{2}}{\tilde{p}_{1} \tilde{p}_{2} \tilde{p}_{3}}
$$


a branch emanating from the origin. From the stability condition (20) the stability of the branch $w_{0}(\omega)$ depends on the sign of $\tilde{p}_{1}$, stable if $\tilde{p}_{1}<0$, unstable if $\tilde{p}_{1}>0$.

When $i=1$, equation (44) becomes

$$
w_{1}(\omega)=\tilde{p}_{1}+i \omega \tilde{\sigma}\left[\tilde{p}_{1} \varepsilon^{1 / 2}\left(\alpha_{1}-\alpha_{0}\right)-\tilde{\sigma}\right] \frac{\left(\tilde{p}_{1}-\tilde{W}_{1}\right)\left(\tilde{p}_{1}-\tilde{W}_{2}\right)}{\tilde{p}_{1}\left(\tilde{p}_{1}-\tilde{p}_{2}\right)\left(\tilde{p}_{1}-\tilde{p}_{3}\right)} .
$$

The stability of $w_{1}(\omega)$ depends on the signs of $\tilde{p}_{1}$ and $\tilde{p}_{1} \varepsilon^{1 / 2}\left(\alpha_{1}-\alpha_{0}\right)-\tilde{\sigma}$. If $\tilde{p}_{1}<0$ then it follows that $\tilde{p}_{1} \varepsilon^{1 / 2}\left(\alpha_{1}-\alpha_{0}\right)-\tilde{\sigma}<0$, so that from inequalities (38) and the stability condition (20), $w_{1}(\omega)$ is unstable. On the other hand, if $\tilde{p}_{1}>0$ then $w_{1}(\omega)$ is stable provided that

$$
\tilde{p}_{1} \varepsilon^{1 / 2}\left(\alpha_{1}-\alpha_{0}\right)-\tilde{\sigma}<0 .
$$

We note that if $\tilde{p}_{1}>0$ then the branch $w_{1}(\omega)$ begins on the positive real axis, to the right of $w_{0}(\omega)$, which begins at the origin. This situation is illustrated in Figure 1 . On the other hand, if $\tilde{p}_{1}<0$ then the branch $w_{1}(\omega)$ begins on the negative real axis, to the left of $w_{0}(\omega)$, as illustrated in Figure 2.

The approximations (45) and (46) both break down in the exceptional case $\tilde{p}_{1}=0$, which is considered later in Section 3.4 and illustrated in Figure 3 .

From inequalities (38) we see that

$$
\frac{\tilde{G}\left(\tilde{p}_{i}\right)}{\tilde{F}^{\prime}\left(\tilde{p}_{i}\right)}>0, \quad i=2,3
$$

whatever the value of $\tilde{p}_{1}$. Therefore the branches $w_{i}(\omega), i=2,3$, are stable only if

$$
\tilde{p}_{i} \varepsilon^{1 / 2}\left(\alpha_{1}-\alpha_{0}\right)-\tilde{\sigma}<0, \quad i=2,3 .
$$

\subsection{High-frequency expansions}

In order to investigate the high-frequency limit we write $\zeta=\omega^{-1}$ and take the limit $\zeta \rightarrow 0$. In terms of $\zeta$ the secular equation (39) becomes

$$
\tilde{F}(w)+\frac{\tilde{\sigma}}{\alpha_{0}\left(1+i \zeta / \alpha_{0}\right)}\left[w \varepsilon^{1 / 2}\left(\alpha_{1}-\alpha_{0}\right)-\tilde{\sigma}\right] \tilde{G}(w)=0 .
$$

For $\zeta=0(\omega \rightarrow \infty)$ the secular equation (50) becomes

$$
H(w) \equiv \tilde{F}(w)+\frac{\tilde{\sigma}}{\alpha_{0}}\left[w \varepsilon^{1 / 2}\left(\alpha_{1}-\alpha_{0}\right)-\tilde{\sigma}\right] \tilde{G}(w)=0 .
$$

$H(w)$ is quartic in $w$ and so has four zeros, denoted by $\bar{h}_{1}, \bar{h}_{2}, \bar{h}_{3}, \bar{h}_{4}$. We determine the sign changes of $H(w)$ using the inequalities (38):

$$
\begin{aligned}
H(-\infty) & =\infty>0 \\
H(0) & =-\alpha_{0}^{-1} \tilde{\sigma}^{2} \tilde{W}_{1} \tilde{W}_{2}<0 \\
H\left(\tilde{W}_{1}\right) & =\tilde{W}_{1}\left(\tilde{W}_{1}-\tilde{p}_{1}\right)\left(\tilde{W}_{1}-\tilde{p}_{2}\right)\left(\tilde{W}_{1}-\tilde{p}_{3}\right)>0 \\
H\left(\tilde{W}_{2}\right) & =\tilde{W}_{2}\left(\tilde{W}_{2}-\tilde{p}_{1}\right)\left(\tilde{W}_{2}-\tilde{p}_{2}\right)\left(\tilde{W}_{2}-\tilde{p}_{3}\right)<0 \\
H(\infty) & =\infty>0 .
\end{aligned}
$$


From the inequalities (52) and (43) we see that the quantities $\bar{h}_{i}$ interlace according to

$$
\bar{h}_{1}<0<\bar{h}_{2}<\tilde{W}_{1}<\bar{h}_{3}<\tilde{W}_{2}<\bar{h}_{4} .
$$

Define a quartic polynomial

$$
\bar{h}(w)=\left(w-\bar{h}_{1}\right)\left(w-\bar{h}_{2}\right)\left(w-\bar{h}_{3}\right)\left(w-\bar{h}_{4}\right),
$$

and observe that $H(w) \equiv \bar{h}(w)$ since both have the same four zeros and both have the same coefficient of $w^{4}$.

For small $\zeta$ the secular equation (50) may be expanded as

$$
\bar{h}(w)-i \zeta \frac{\tilde{\sigma}}{\alpha_{0}^{2}}\left[w \varepsilon^{1 / 2}\left(\alpha_{1}-\alpha_{0}\right)-\tilde{\sigma}\right] \tilde{G}(w)=0,
$$

neglecting terms $O\left(\zeta^{2}\right)$. Taylor expansions of the four roots of equation (55) in the highfrequency limit, reverting to the notation $\zeta=\omega^{-1}$, take the form

$$
w_{i}(\omega)=\bar{h}_{i}+i \omega^{-1} \frac{\tilde{\sigma}}{\alpha_{0}^{2}}\left[\bar{h}_{i} \varepsilon^{1 / 2}\left(\alpha_{1}-\alpha_{0}\right)-\tilde{\sigma}\right] \frac{\tilde{G}\left(\bar{h}_{i}\right)}{\bar{h}^{\prime}\left(\bar{h}_{i}\right)}, \quad i=1,2,3,4,
$$

at high frequency. From the inequalities (53), we find that

$$
\frac{\tilde{G}\left(\bar{h}_{1}\right)}{\bar{h}^{\prime}\left(\bar{h}_{1}\right)}<0, \quad \frac{\tilde{G}\left(\bar{h}_{i}\right)}{\bar{h}^{\prime}\left(\bar{h}_{i}\right)}>0, \quad i=2,3,4 .
$$

Consider first the branch $i=1$ of equation (56). Since $\bar{h}_{1}<0$ it follows that

$$
\bar{h}_{1} \varepsilon^{1 / 2}\left(\alpha_{1}-\alpha_{0}\right)-\tilde{\sigma}<0
$$

and so from equation $(57)_{1}$ we see that $\operatorname{Im} w_{1}(\omega)>0$ and so $w_{1}(\omega)$ is unstable at high frequency. From equation $(57)_{2}$ we see that the stability of the other branches $w_{i}(\omega), i=$ $2,3,4$, is determined by the sign of

$$
\bar{h}_{i} \varepsilon^{1 / 2}\left(\alpha_{1}-\alpha_{0}\right)-\tilde{\sigma}, \quad i=2,3,4,
$$

stable if negative, unstable if positive.

To show that the branches cannot change their stability nature at intermediate frequencies we rewrite the secular equation (39) in the form

$$
\frac{\tilde{F}(w)}{\left[w \varepsilon^{1 / 2}\left(\alpha_{1}-\alpha_{0}\right)-\tilde{\sigma}\right] \tilde{G}(w)}=\frac{i \omega \tilde{\sigma}}{1-i \omega \alpha_{0}} .
$$

Branches can only change their stability nature at a real value of $w$ because the real axis forms the boundary between the stable region $\operatorname{Im} w(\omega)<0$ and the unstable region $\operatorname{Im} w(\omega)>0$. We observe that for no intermediate frequency $0<\omega<\infty$ and real $w$ can equation (60) be satisfied. It follows that branches cannot change their stability nature at intermediate frequencies. 


\subsection{The exceptional case $\tilde{p}_{1}=0$.}

When $\tilde{p}_{1}=0$ the secular equation (39), with the help of equation (40), becomes

$$
w^{2}\left(w-\tilde{p}_{2}\right)\left(w-\tilde{p}_{3}\right)-\frac{i \omega \tilde{\sigma}}{1-i \omega \alpha_{0}}\left[w \varepsilon^{1 / 2}\left(\alpha_{1}-\alpha_{0}\right)-\tilde{\sigma}\right]\left(w-\tilde{W}_{1}\right)\left(w-\tilde{W}_{2}\right)=0 .
$$

For small $\omega$ we seek solutions of the form $w=A(i \omega)^{n}$ where $A$ and $n$ are constants to be determined. We find that $n=1 / 2$ and that the two resulting branches of $w$, both emanating from the origin, are approximated for small $\omega$ by

$$
w_{0,1}(\omega)=\mp e^{-\pi i / 4} \omega^{1 / 2} \tilde{\sigma}\left(\frac{\tilde{W}_{1} \tilde{W}_{2}}{\tilde{p}_{2} \tilde{p}_{3}}\right)^{1 / 2}
$$

the first with argument $3 \pi / 4$, and so unstable, and the second with argument $-\pi / 4$, and so stable. This situation is illustrated in Figure 3.

\subsection{Numerical results}

Figure 1 illustrates the case $\tilde{p}_{1}>0$. The branch $w_{0}(\omega)$ beginning at the origin is unstable in each part of the Figure. If $\alpha_{1}-\alpha_{0}$ is small enough then

$$
\tilde{p}_{i} \varepsilon^{1 / 2}\left(\alpha_{1}-\alpha_{0}\right)-\tilde{\sigma}<0, \quad \text { for } \quad i=1,2,3,
$$

at low frequencies and

$$
\bar{h}_{i} \varepsilon^{1 / 2}\left(\alpha_{1}-\alpha_{0}\right)-\tilde{\sigma}<0, \quad \text { for } \quad i=1,2,3,
$$

at high frequencies. Therefore, the branches $w_{i}(\omega), i=1,2,3$, are stable if $\alpha_{1}-\alpha_{0}$ is small enough. This is clear in the first subfigures (a)-(c) of Figure 1 where $\alpha_{1}-\alpha_{0}$ is small. As $\alpha_{1}-\alpha_{0}$ increases, first $w_{3}(\omega)$ becomes unstable, see part $(\mathrm{d})$, and as it increases further other branches become unstable.

Figure 2 illustrates the case $\tilde{p}_{1}<0$. The left hand branch, beginning at $w=\tilde{p}_{1}$, is unstable in each part of the Figure and, as with Figure 1, more branches become unstable as $\alpha_{0}$ and $\alpha_{1}$ increase. In contrast with Figure 1, the branch $w_{0}(\omega)$ beginning at the origin is stable in each part of the Figure.

Figure 3 illustrates the exceptional case $\tilde{p}_{1}=0$ and we see two branches beginning at the origin, one stable with argument $-\pi / 4$, the other unstable with argument $-3 \pi / 4$. As with Figure 1, more branches become unstable as $\alpha_{0}$ and $\alpha_{1}$ increase. 


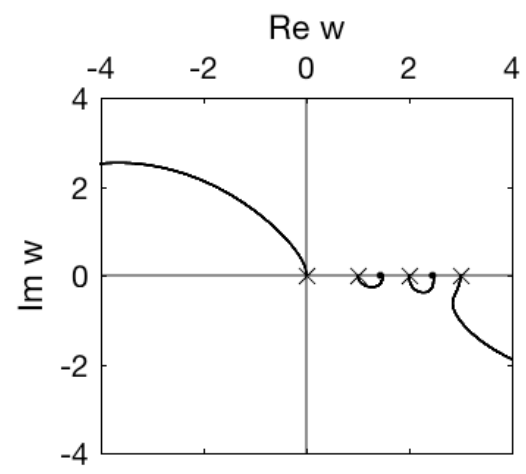

(a) $\alpha_{0}=0.02, \alpha_{1}=0.03$

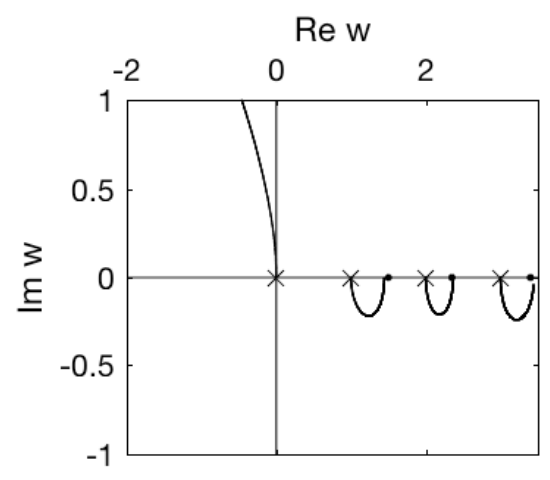

(c) $\alpha_{0}=0.07, \alpha_{1}=0.3$

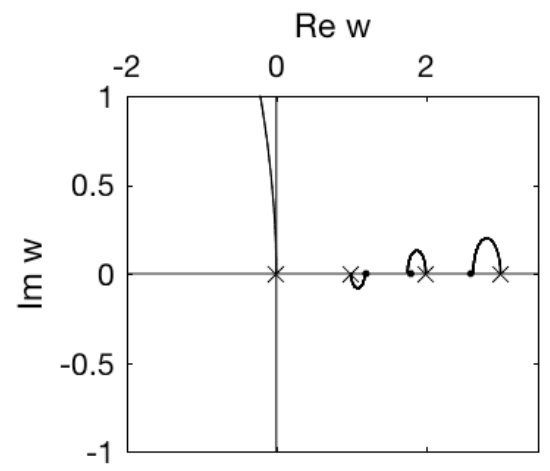

(e) $\alpha_{0}=0.15, \alpha_{1}=0.9$

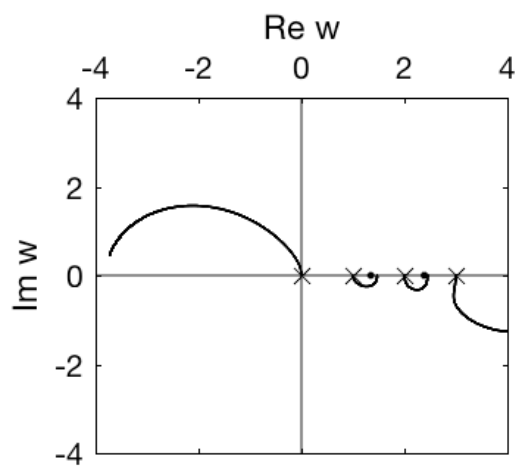

(b) $\alpha_{0}=0.05, \alpha_{1}=0.07$

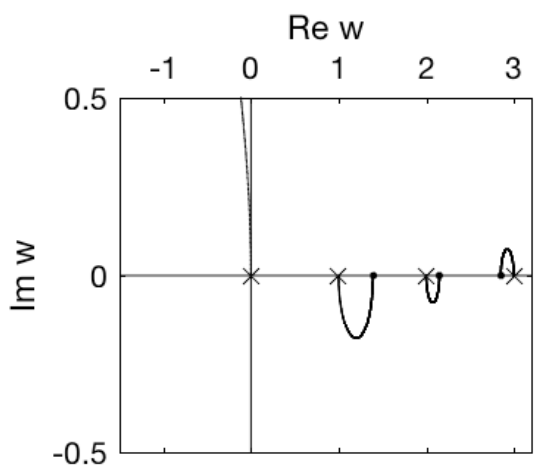

(d) $\alpha_{0}=0.1, \alpha_{1}=0.5$

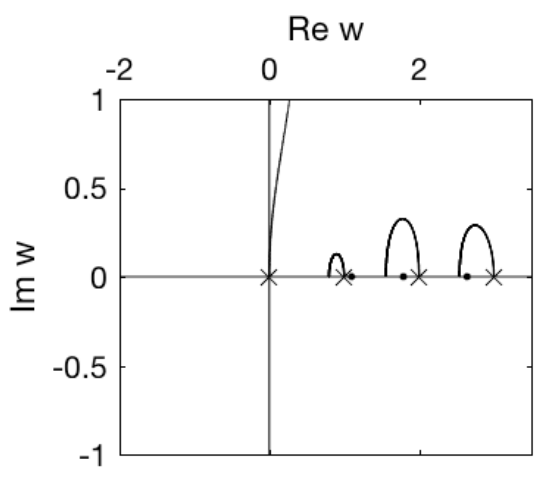

(f) $\alpha_{0}=0.1, \alpha_{1}=1.4$

Figure 1: The squared wave speeds of constrained anisotropic temperature-rate-dependent thermoelasticity. For each part, $\tilde{p}_{1}=1, \tilde{p}_{2}=2, \tilde{p}_{3}=3, \tilde{W}_{1}=1.5, \tilde{W}_{2}=2.5, \tilde{\sigma}=1, \varepsilon=1$. 


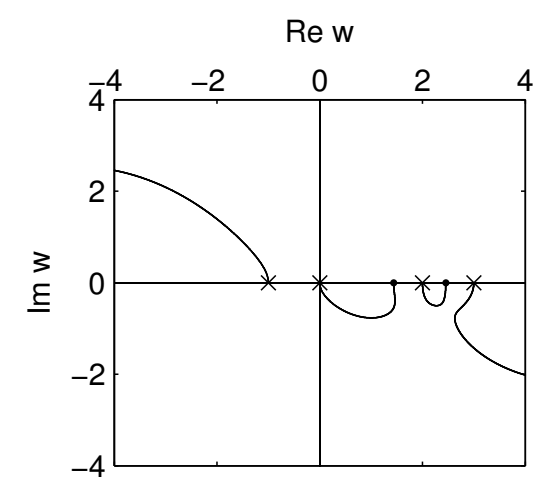

(a) $\alpha_{0}=0.02, \alpha_{1}=0.03$

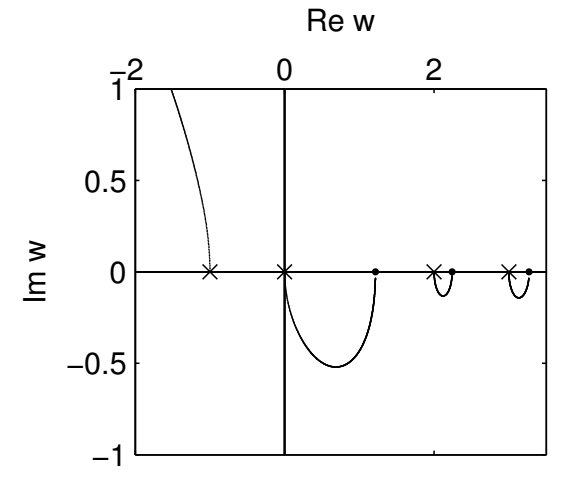

(c) $\alpha_{0}=0.07, \alpha_{1}=0.3$

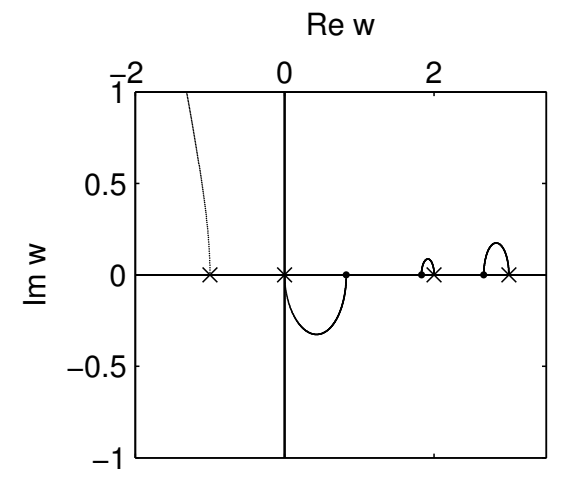

(e) $\alpha_{0}=0.1, \alpha_{1}=0.9$

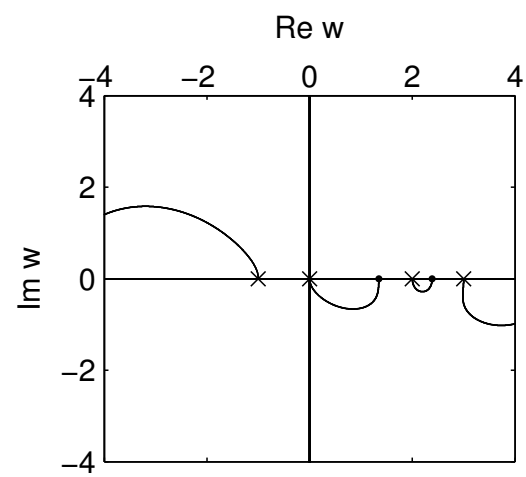

(b) $\alpha_{0}=0.05, \alpha_{1}=0.07$

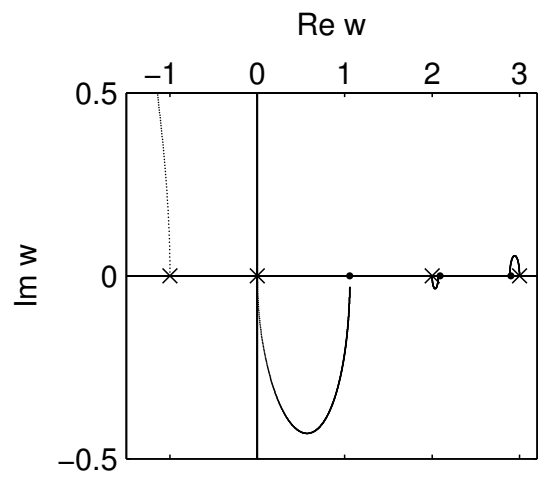

(d) $\alpha_{0}=0.1, \alpha_{1}=0.5$

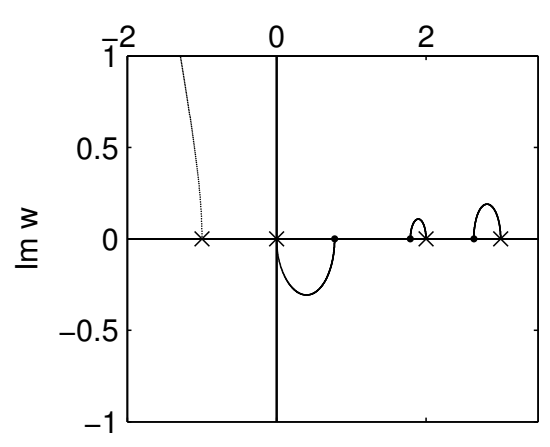

(f) $\alpha_{0}=0.1, \alpha_{1}=1$

Figure 2: The squared wave speeds of constrained anisotropic temperature-rate-dependent thermoelasticity. For each part, $\tilde{p}_{1}=-1, \tilde{p}_{2}=2, \tilde{p}_{3}=3, \tilde{W}_{1}=1.5, \tilde{W}_{2}=2.5, \tilde{\sigma}=1, \varepsilon=1$. 


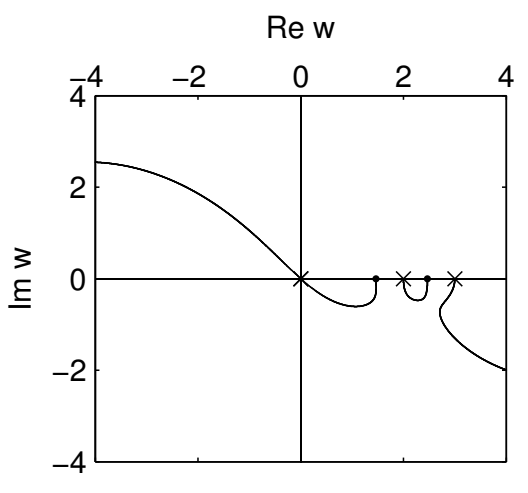

(a) $\alpha_{0}=0.02, \alpha_{1}=0.03$

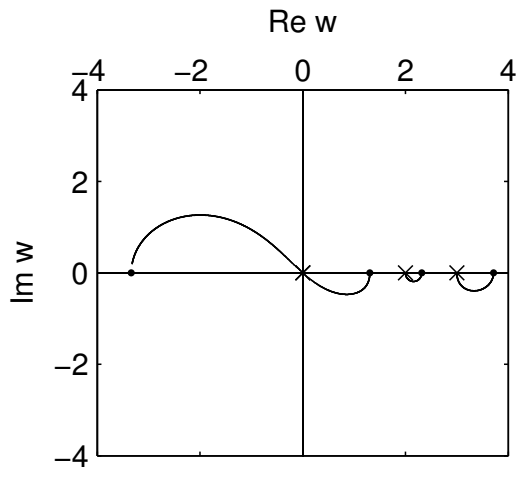

(c) $\alpha_{0}=0.1, \alpha_{1}=0.2$

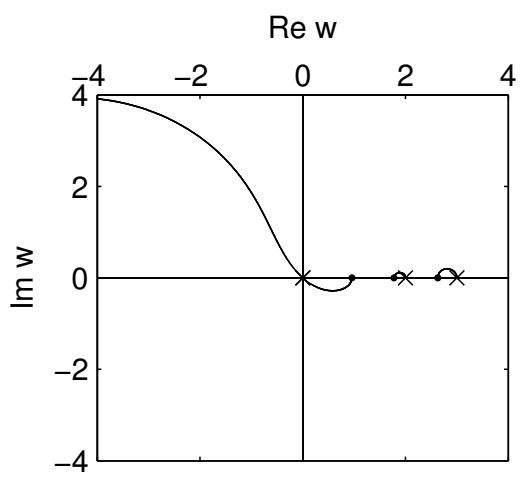

(e) $\alpha_{0}=0.1, \alpha_{1}=0.9$

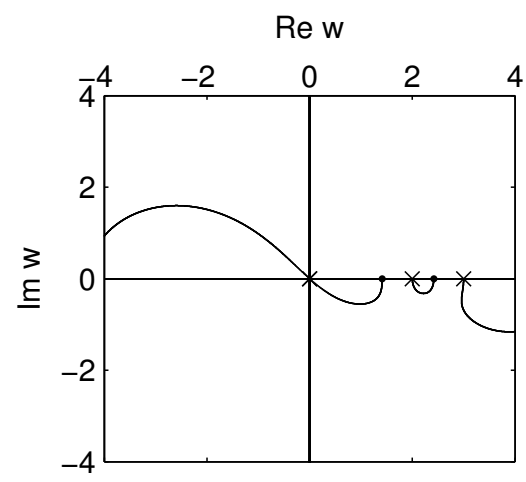

(b) $\alpha_{0}=0.05, \alpha_{1}=0.07$

Rew

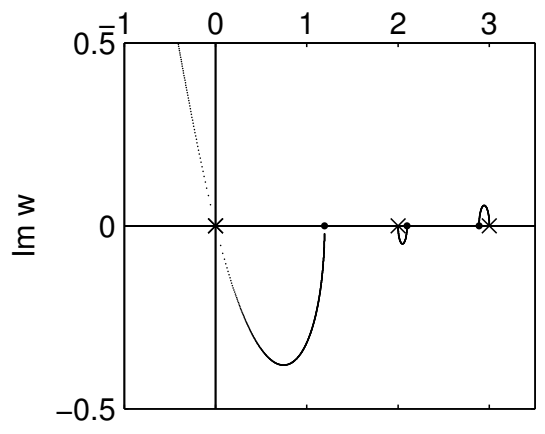

(d) $\alpha_{0}=0.1, \alpha_{1}=0.5$

Re w

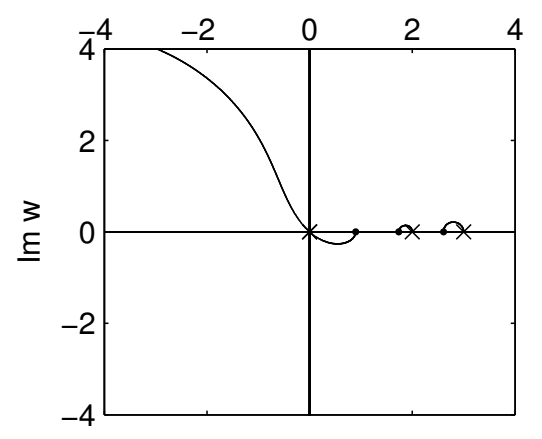

(f) $\alpha_{0}=0.1, \alpha_{1}=1$

Figure 3: The squared wave speeds of constrained anisotropic temperature-rate-dependent thermoelasticity. For each part, $\tilde{p}_{1}=0, \tilde{p}_{2}=2, \tilde{p}_{3}=3, \tilde{W}_{1}=1.5, \tilde{W}_{2}=2.5, \tilde{\sigma}=1, \varepsilon=1$. 


\section{Constrained isotropic temperature-rate-dependent thermoelasticity}

In order to find the secular equation for constrained isotropic temperature-rate-dependent thermoelasticity we specialize the results of the previous section in the anisotropic case following the methods of Alharbi and Scott [20] in the unconstrained case.

\subsection{The secular equation}

For a material that is both mechanically and thermally isotropic, and suffers a constraint of incompressibility at constant temperature, we have

$$
\tilde{c}_{i j k l}=\lambda^{\prime} \delta_{i j} \delta_{k l}+\mu^{\prime}\left(\delta_{i k} \delta_{j l}+\delta_{i l} \delta_{j k}\right), \quad \beta_{i j}=\beta \delta_{i j}, \quad k_{i j}=k \delta_{i j}, \quad \tilde{N}_{i j}=\delta_{i j},
$$

where $\lambda^{\prime}$ and $\mu^{\prime}$ are the Lamé moduli, $\beta$ is the scalar temperature coefficient of stress and $k$ is the scalar thermal conductivity, leading to $\tilde{\mathbf{c}}=\mathbf{b}=\mathbf{n}$, as before. We define dimensionless Lamé moduli by

$$
\lambda=\lambda^{\prime} / \gamma, \quad \mu=\mu^{\prime} / \gamma,
$$

where the parameter $\gamma$ will shortly be prescribed. From equation $(63)_{1}$ we see that the isothermal acoustic tensor $(22)_{1}$ reduces to

$$
\tilde{\mathbf{Q}}=\mu \mathbf{1}+(\lambda+\mu) \mathbf{n} \otimes \mathbf{n}
$$

in the isotropic case and in this case $\mathbf{M}$ defined by equations (27)-(29) reduces to

$$
\mathbf{M}=(\mu-w) \mathbf{1}+[\lambda+\mu+K] \mathbf{n} \otimes \mathbf{n},
$$

where $K$ is defined by

$$
K=\varepsilon-\left(\varepsilon^{1 / 2}+\tilde{\sigma}\right)^{2}+\frac{i \omega \tilde{\sigma}}{1-i \omega \alpha_{0}}\left[\varepsilon^{1 / 2}\left(\alpha_{1}-\alpha_{0}\right)-\frac{\tilde{\sigma}}{w}\right] .
$$

An application of the identity (32) to the tensor $\mathbf{M}$ defined by equation (65) gives the secular equation $\operatorname{det} \mathbf{M}=0$ in the form

$$
\operatorname{det}\{(\mu-w) \mathbf{1}\}+[\lambda+\mu+K] \mathbf{n} \cdot\{(\mu-w) \mathbf{1}\}^{\text {adj }} \mathbf{n}=0
$$

Because

$$
\operatorname{det}\{(\mu-w) \mathbf{1}\}=(\mu-w)^{3} \text { and } \mathbf{n} \cdot\{(\mu-w) \mathbf{1}\}^{\mathrm{adj}} \mathbf{n}=(\mu-w)^{2}
$$

we can rewrite equation (67) in the form

$$
(\mu-w)^{2}[\lambda+2 \mu-w+K]=0 .
$$

We now select a convenient value for the scaling parameter $\gamma$, namely,

$$
\gamma=\lambda^{\prime}+2 \mu^{\prime}, \quad \text { so that } \quad \lambda+2 \mu=1,
$$


and the secular equation (69) becomes in fully non-dimensional form

$$
(w-\mu)^{2}[1-w+K]=0 .
$$

The choice $(70)_{1}$ of scaling parameter means that we are comparing all squared wave speeds with the squared wave speed $\left(\lambda^{\prime}+2 \mu^{\prime}\right) / \rho$ of purely elastic longitudinal waves in an isothermal isotropic linearly elastic solid so that the dimensionless isothermal longitudinal squared wave speed is given by $w=1$.

The secular equation (71) has the repeated root

$$
w=\mu=\frac{\mu^{\prime}}{\lambda^{\prime}+2 \mu^{\prime}}
$$

which corresponds to two transverse non-dispersive purely elastic isothermal isotropic waves unaffected by heat conduction. Since usually $\lambda^{\prime}>0$ the dimensionless transverse squared wave speed $w=\mu$ is restricted by

$$
0<\mu<\frac{1}{2}
$$

as compared with the dimensionless isothermal longitudinal squared wave speed $w=1$.

The roots corresponding to the longitudinal waves are given by the remaining factor of (71):

$$
w-1-K=0 .
$$

On using the definition (66), multiplying throughout by $w$, and rearranging, we see that the final form of the dimensionless secular equation for longitudinal waves in isotropic constrained temperature-rate-dependent thermoelasticity is given by

$$
w\left(w-\tilde{p}_{1}\right)-\frac{i \omega \tilde{\sigma}}{1-i \omega \alpha_{0}}\left[w \varepsilon^{1 / 2}\left(\alpha_{1}-\alpha_{0}\right)-\tilde{\sigma}\right]=0
$$

in which $\tilde{p}_{1}$ is defined by

$$
\begin{aligned}
\tilde{p}_{1} & =1+\varepsilon-\left(\varepsilon^{1 / 2}+\tilde{\sigma}\right)^{2}, \\
& =1-2 \varepsilon^{1 / 2} \tilde{\sigma}-\tilde{\sigma}^{2}, \\
& =-\left(\tilde{\sigma}-\tilde{\sigma}_{c}\right)\left[(1+\varepsilon)^{1 / 2}+\varepsilon^{1 / 2}\right] .
\end{aligned}
$$

We see that $\tilde{p}_{1}=0$ only when $\tilde{\sigma}$ takes the critical value $\tilde{\sigma}_{c}$ defined by

$$
\tilde{\sigma}_{c}=(1+\varepsilon)^{1 / 2}-\varepsilon^{1 / 2}
$$

so that $0<\tilde{\sigma}_{c}<1$. If $\tilde{\sigma}>\tilde{\sigma}_{c}$ then $\tilde{p}_{1}<0$ and if $\tilde{\sigma}<\tilde{\sigma}_{c}$ then $\tilde{p}_{1}>0$.

The isotropic secular equation (74) is consistent with the anisotropic form (39) of the secular equation if we take

$$
\tilde{p}_{2}=\tilde{p}_{3}=\tilde{W}_{1}=\tilde{W}_{2}=\mu
$$

in that equation and allow $\tilde{p}_{1}$ to be defined by equation $(75)$. 


\subsection{Low-frequency expansions}

When $\omega=0$, the roots of the secular equation (74) are given by $w=\tilde{p}_{i}, i=0$, 1 , defining $\tilde{p}_{0} \equiv 0$ and $\tilde{p}_{1}$ by equation (75). When $\omega \rightarrow 0$, the two roots of the secular equation may be obtained as Taylor expansions of equation (74):

$$
w_{0}(\omega)=\frac{i \omega \tilde{\sigma}^{2}}{\tilde{p}_{1}}, \quad w_{1}(\omega)=\tilde{p}_{1}+i \omega \tilde{\sigma}\left[\varepsilon^{1 / 2}\left(\alpha_{1}-\alpha_{0}\right)-\frac{\tilde{\sigma}}{\tilde{p}_{1}}\right] .
$$

From the stability condition $(20)$ the stability of the branch $w_{0}(\omega)$ depends on the sign of $\tilde{p}_{1}$, stable if $\tilde{p}_{1}<0$, unstable if $\tilde{p}_{1}>0$. The stability of $w_{1}(\omega)$ depends on the signs of $\tilde{p}_{1}$ and $\varepsilon^{1 / 2}\left(\alpha_{1}-\alpha_{0}\right)-\tilde{\sigma} / \tilde{p}_{1}$. If $\tilde{p}_{1}<0$ then $\varepsilon^{1 / 2}\left(\alpha_{1}-\alpha_{0}\right)-\tilde{\sigma} / \tilde{p}_{1}>0$, so that $w_{1}(\omega)$ is unstable. On the other hand, if $\tilde{p}_{1}>0$ then $w_{1}(\omega)$ is stable provided that

$$
\varepsilon^{1 / 2}\left(\alpha_{1}-\alpha_{0}\right)-\tilde{\sigma} / \tilde{p}_{1}<0
$$

The approximations $(78)_{1,2}$ both break down when $\tilde{p}_{1}=0$. In this exceptional case, the approximations $(62)$ reduce to

$$
w_{0,1}(\omega)=\mp e^{-i \pi / 4} \omega^{1 / 2} \tilde{\sigma}_{c}
$$

for isotropic materials on account of the equalities (77). As before, the first has argument $3 \pi / 4$, and so is unstable, and the second has argument $-\pi / 4$, and so is stable and both branches meet at the origin.

\subsection{High-frequency expansions}

In order to investigate the high-frequency limit we write $\zeta=\omega^{-1}$ and take the limit $\zeta \rightarrow 0$. In terms of $\zeta$ the secular equation (39) becomes

$$
w\left(w-\tilde{p}_{1}\right)+\frac{\tilde{\sigma}}{\alpha_{0}\left(1+i \zeta / \alpha_{0}\right)}\left[w \varepsilon^{1 / 2}\left(\alpha_{1}-\alpha_{0}\right)-\tilde{\sigma}\right]=0 .
$$

For $\zeta=0(\omega \rightarrow \infty)$ the secular equation (81) becomes

$$
H(w)=w\left(w-\tilde{p}_{1}\right)+\frac{\tilde{\sigma}}{\alpha_{0}}\left[w \varepsilon^{1 / 2}\left(\alpha_{1}-\alpha_{0}\right)-\tilde{\sigma}\right]=0 .
$$

To determine the positions of zeros of $H(w)$ we need to examine its sign changes:

$$
\begin{aligned}
H(-\infty) & =\infty>0, \\
H(0) & =-\tilde{\sigma}^{2} / \alpha_{0}<0, \\
H(\infty) & =\infty>0 .
\end{aligned}
$$

So the two real roots $\bar{h}_{1}$ and $\bar{h}_{2}$ of $H(w)=0$ satisfy

$$
\bar{h}_{1}<0<\bar{h}_{2} .
$$


They are the roots of the quadratic polynomial $\bar{h}(w)=\left(w-\bar{h}_{1}\right)\left(w-\bar{h}_{2}\right)$ and we must have $H(w)=\bar{h}(w)$.

For small $\zeta$ the secular equation (81) may be expanded as

$$
\bar{h}(w)-i \zeta \frac{\tilde{\sigma}}{\alpha_{0}^{2}}\left[w \varepsilon^{1 / 2}\left(\alpha_{1}-\alpha_{0}\right)-\tilde{\sigma}\right]=0 .
$$

Taylor expansions of the two roots of this equation in the high-frequency limit, reverting to the notation $\zeta=\omega^{-1}$, take the forms

$$
w_{1}=\bar{h}_{1}-i \omega^{-1} \frac{\tilde{\sigma}}{\alpha_{0}^{2}} \frac{\bar{h}_{1} \varepsilon^{1 / 2}\left(\alpha_{1}-\alpha_{0}\right)-\tilde{\sigma}}{\bar{h}_{2}-\bar{h}_{1}}, \quad w_{2}=\bar{h}_{2}+i \omega^{-1} \frac{\tilde{\sigma}}{\alpha_{0}^{2}} \frac{\bar{h}_{2} \varepsilon^{1 / 2}\left(\alpha_{1}-\alpha_{0}\right)-\tilde{\sigma}}{\bar{h}_{2}-\bar{h}_{1}} .
$$

From equation (82), $\bar{h}_{2}-\bar{h}_{1}>0$ and $\bar{h}_{1}<0$ and so $w_{1}$ is unstable. Also, $w_{2}$ is unstable unless

$$
\bar{h}_{2} \varepsilon^{1 / 2}\left(\alpha_{1}-\alpha_{0}\right)-\tilde{\sigma}<0
$$

\subsection{Numerical results}

In each of Figures 4 and 5 we take $\alpha_{0}=0.01$ and $\alpha_{1}=0.02$ and we choose $\varepsilon=0$ in Figure 4 and $\varepsilon=1$ in Figure 5. In both figures we have two longitudinal waves, one stable and the other unstable. If $\tilde{\sigma}<\tilde{\sigma}_{c}$ the stable branch starts from the point $w=\tilde{p}_{1}$, where $\tilde{p}_{1}$ is defined by equation (75), and the unstable branch begins at the origin, see subfigures (a) and (b). But this situation is reversed if $\tilde{\sigma}>\tilde{\sigma}_{c}$ as shown in subfigures (d)-(f). In the special case when $\tilde{\sigma}=\tilde{\sigma}_{c}$ the branches become a connected line passing through the origin at angle $-\pi / 4$ to the real axis, see subfigure (c), as predicted by equation (80). 
(a) $\tilde{\sigma}=0.1 \tilde{\sigma}_{c}$

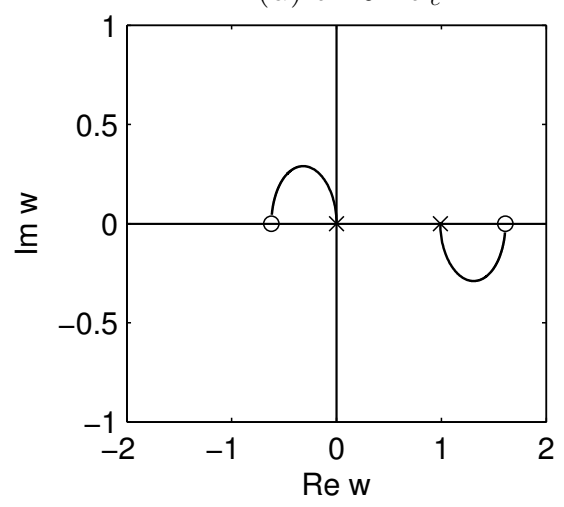

(c) $\tilde{\sigma}=\tilde{\sigma}_{c}$

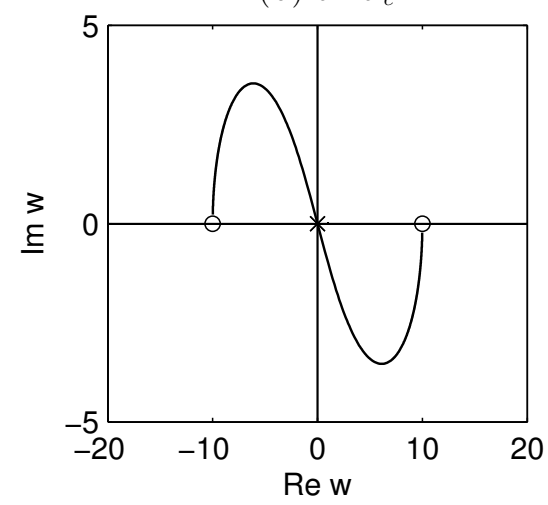

(e) $\tilde{\sigma}=2 \tilde{\sigma}_{c}$

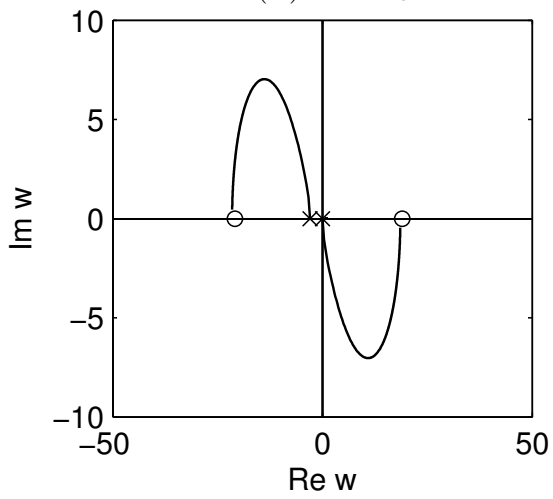

(b) $\tilde{\sigma}=0.6 \tilde{\sigma}_{c}$

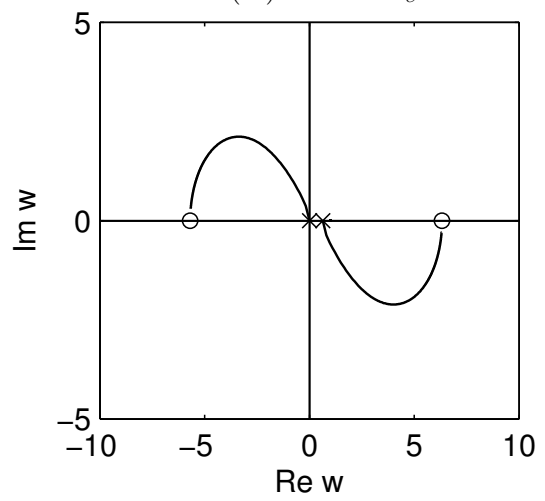

(d) $\tilde{\sigma}=1.3 \tilde{\sigma}_{c}$

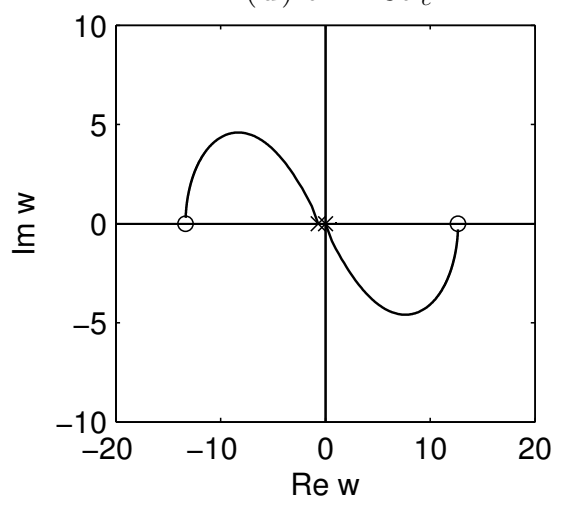

(f) $\tilde{\sigma}=10 \tilde{\sigma}_{c}$

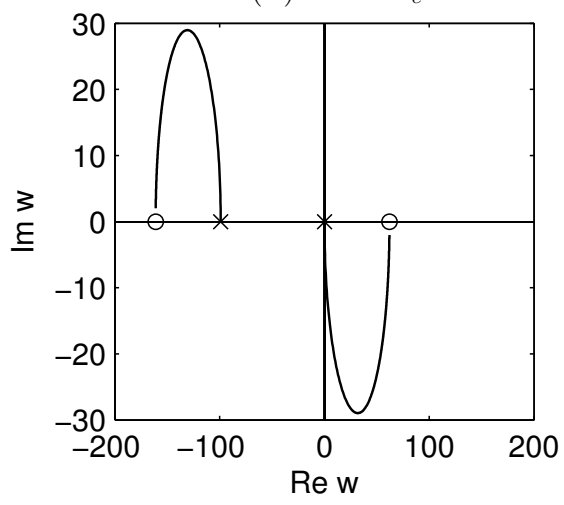

Figure 4: The longitudinal squared wave speeds of constrained isotropic temperature-ratedependent thermoelasticity. For each part, $\varepsilon=0, \alpha_{0}=0.01, \alpha_{1}=0.02$. 
(a) $\tilde{\sigma}=0.1 \tilde{\sigma}_{c}$

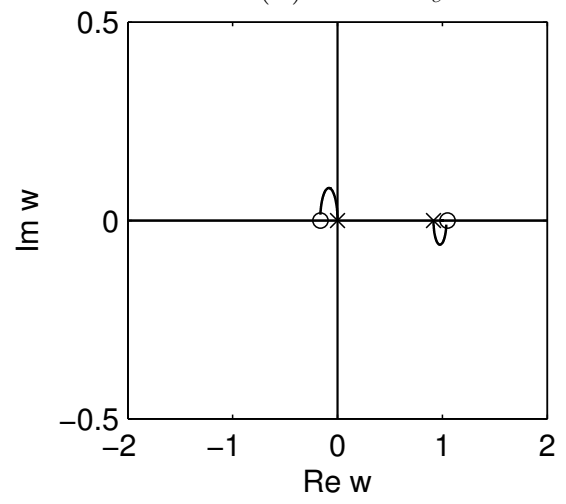

(c) $\tilde{\sigma}=\tilde{\sigma}_{c}$

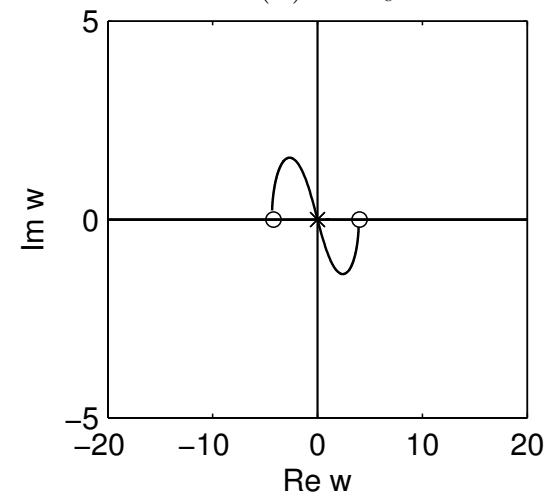

(e) $\tilde{\sigma}=2 \tilde{\sigma}_{c}$

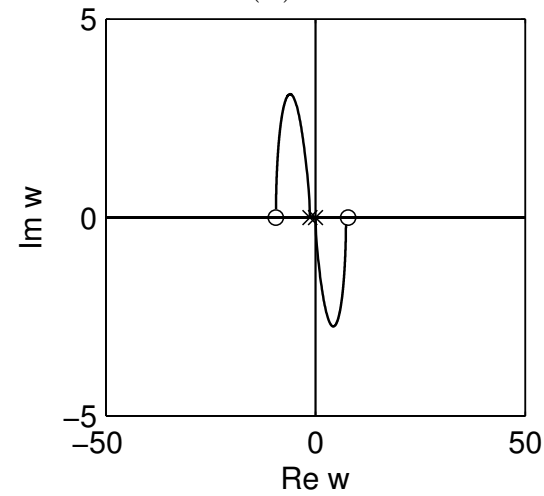

(b) $\tilde{\sigma}=0.6 \tilde{\sigma}_{c}$

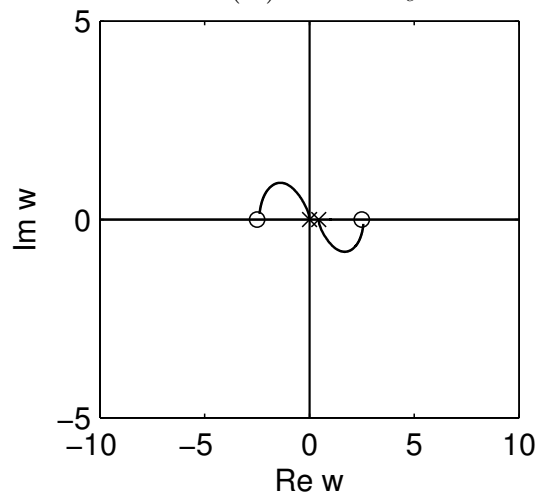

(d) $\tilde{\sigma}=1.3 \tilde{\sigma}_{c}$

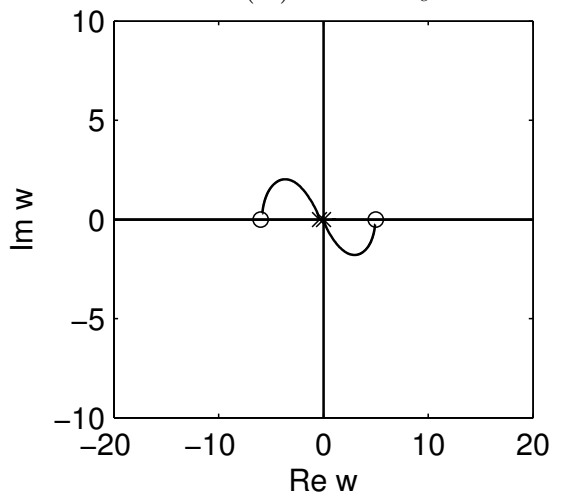

(f) $\tilde{\sigma}=10 \tilde{\sigma}_{c}$

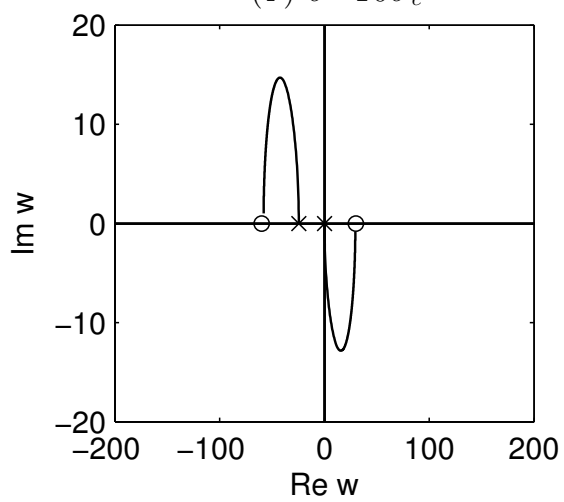

Figure 5: The longitudinal squared wave speeds of constrained isotropic temperature-ratedependent thermoelasticity. For each part, $\varepsilon=1, \alpha_{0}=0.01, \alpha_{1}=0.02$. 


\section{Constrained anisotropic generalized temperature-rate- dependent thermoelasticity}

We are concerned in this section with the theory of temperature-rate-dependent thermoelasticity combined with generalized thermoelasticity and subjected to a deformationtemperature constraint. The relevant field equations have been derived at equation (18). Again, we follow the method of Alharbi and Scott [21] and also the analysis of Section 3.

\subsection{The secular equation}

We insert the plane harmonic wave forms (19) into the field equations (18) in order to obtain the equations

$$
\begin{aligned}
\left(\tilde{Q}_{i k}-w \delta_{i k}\right) U_{k}-(i \omega s)^{-1} \gamma^{-1} b_{i}\left(1-i \omega \alpha_{1}\right) \Theta+(i \omega s)^{-1} \gamma^{-1} \tilde{c}_{i} \tilde{H} & =0, \\
(i \omega s) T b_{k} U_{k}+\left[\frac{(i \omega s) s k}{1-i \omega \tau}+\rho c\left(1-i \omega \alpha_{0}\right)\right] \Theta+\alpha T \tilde{H} & =0, \\
(i \omega s) \tilde{c}_{k} U_{k}-\alpha\left(1-i \omega \alpha_{0}\right) \Theta & =0,
\end{aligned}
$$

of generalized temperature-rate-dependent thermoelasticity, the counterpart of those obtained at equation (21) in the non-generalized case. We see that equation $(83)_{2}$ differs from equation $(21)_{2}$ by the factor $1-i \omega \tau$ in the denominator of the second term but otherwise the equations are the same.

We now eliminate $\Theta$ and $\tilde{H}$ between equations (83) as in Section 3 and by nondimensionalizing, simplifying and rearranging the resulting equation we finally obtain

$$
\tilde{F}(w)-\frac{i \omega \tilde{\sigma}}{1-i \omega \alpha_{0}}\left[w \varepsilon^{1 / 2}\left(\alpha_{1}-\alpha_{0}\right)-\frac{\tilde{\sigma}}{1-i \omega \tau}\right] \tilde{G}(w)=0,
$$

where $\tilde{F}(w)$ and $\tilde{G}(w)$ are defined at equation (40). Putting $\tau=0$ we get the familiar secular equation (39) of temperature-rate-dependent thermoelasticity.

Equation (84) is the secular equation for anisotropic generalized temperature-ratedependent thermoelasticity which is constrained by a deformation-temperature constraint.

\subsection{Low-frequency expansions}

When $\omega \rightarrow 0$ the relaxation time $\tau$ in equation (84) does not appear in the solutions so the low-frequency behaviour is exactly as in Section 3.2.

Summarizing, the branch $w_{0}(\omega)$ is stable if $\tilde{p}_{1}<0$, and unstable if $\tilde{p}_{1}>0$, while the branches $w_{i}(\omega), i=1,2,3$, are stable in the low-frequency regimes if the quantity

$$
\tilde{p}_{i} \varepsilon^{1 / 2}\left(\alpha_{1}-\alpha_{0}\right)-\tilde{\sigma}
$$

is negative. Conversely, these branches are unstable if this quantity is positive.

In the exceptional case $\tilde{p}_{1}=0$, the approximations (62) once again hold. 


\subsection{High-frequency expansions}

The roots of the secular equation in the high-frequency limit $\omega \rightarrow \infty$, may be obtained by putting $\omega=\zeta^{-1}$, so the secular equation (84) becomes

$$
\tilde{F}(w)+\frac{\tilde{\sigma}}{\alpha_{0}\left(1+i \zeta / \alpha_{0}\right)}\left[w \varepsilon^{1 / 2}\left(\alpha_{1}-\alpha_{0}\right)-\frac{i \zeta \tilde{\sigma}}{\tau(1+i \zeta / \tau)}\right] \tilde{G}(w)=0 .
$$

Putting $\zeta=0$, we obtain

$$
H \equiv \tilde{F}(w)+\frac{\tilde{\sigma}}{\alpha_{0}} w \varepsilon^{1 / 2}\left(\alpha_{1}-\alpha_{0}\right) \tilde{G}(w)=0 .
$$

$H$ is a quartic in $w$ so there are four roots with one root $w=0$ denoted by $\bar{h}_{1}=0$ and the others by $\bar{h}_{i}, i=2,3,4$. We examine the sign changes of $H$ using the inequalities (38):

$$
\begin{aligned}
H(-\infty) & =\infty>0, \\
H(0) & =0 \\
H\left(\tilde{p}_{1}\right) & =\tilde{\sigma} \tilde{p}_{1} \varepsilon^{1 / 2} \alpha_{0}^{-1}\left(\alpha_{1}-\alpha_{0}\right)\left(\tilde{p}_{1}-\tilde{W}_{1}\right)\left(\tilde{p}_{1}-\tilde{W}_{2}\right), \\
H\left(\tilde{W}_{1}\right) & =\tilde{W}_{1}\left(\tilde{W}_{1}-\tilde{p}_{1}\right)\left(\tilde{W}_{1}-\tilde{p}_{2}\right)\left(\tilde{W}_{1}-\tilde{p}_{3}\right)>0 \\
H\left(\tilde{p}_{2}\right) & =\tilde{\sigma} \tilde{p}_{2} \varepsilon^{1 / 2} \alpha_{0}^{-1}\left(\alpha_{1}-\alpha_{0}\right)\left(\tilde{p}_{2}-\tilde{W}_{1}\right)\left(\tilde{p}_{2}-\tilde{W}_{2}\right)<0, \\
H\left(\tilde{W}_{2}\right) & =\tilde{W}_{2}\left(\tilde{W}_{2}-\tilde{p}_{1}\right)\left(\tilde{W}_{2}-\tilde{p}_{2}\right)\left(\tilde{W}_{2}-\tilde{p}_{3}\right)<0 \\
H\left(\tilde{p}_{3}\right) & =\tilde{\sigma} \tilde{p}_{3} \varepsilon^{1 / 2} \alpha_{0}^{-1}\left(\alpha_{1}-\alpha_{0}\right)\left(\tilde{p}_{3}-\tilde{W}_{1}\right)\left(\tilde{p}_{3}-\tilde{W}_{2}\right)>0 \\
H(\infty) & =\infty>0 .
\end{aligned}
$$

From these inequalities we see that if $\tilde{p}_{1}>0$ the roots $\bar{h}_{i}$ satisfy the inequalities

$$
\bar{h}_{2}<\tilde{p}_{1}<\tilde{W}_{1}<\bar{h}_{3}<\tilde{p}_{2}<\tilde{W}_{2}<\bar{h}_{4}<\tilde{p}_{3}
$$

and if $\tilde{p}_{1}<0$, they satisfy

$$
\bar{h}_{2}<\tilde{p}_{1}<0=\bar{h}_{1}<\tilde{W}_{1}<\bar{h}_{3}<\tilde{p}_{2}<\tilde{W}_{2}<\bar{h}_{4}<\tilde{p}_{3} .
$$

In either case we define a quartic polynomial

$$
\bar{h}(w)=w\left(w-\bar{h}_{2}\right)\left(w-\bar{h}_{3}\right)\left(w-\bar{h}_{4}\right),
$$

and observe that $H(w) \equiv \bar{h}(w)$.

Now looking for roots when $\zeta \rightarrow 0$, equation (85) is well approximated by

$$
\bar{h}(w)-i \zeta \frac{\tilde{\sigma}}{\alpha_{0}}\left[w \varepsilon^{1 / 2} \alpha_{0}^{-1}\left(\alpha_{1}-\alpha_{0}\right)+\frac{\tilde{\sigma}}{\tau}\right] \tilde{G}(w)=0 .
$$

This equation is quartic in $w$, so there are four roots $w_{i}, i=1,2,3,4$. Power series expansion of the roots of the secular equation in the high-frequency limit take the form

$$
w_{i}(\omega)=\bar{h}_{i}+i \omega^{-1} \frac{\tilde{\sigma}}{\alpha_{0}}\left[\bar{h}_{i} \varepsilon^{1 / 2} \alpha_{0}^{-1}\left(\alpha_{1}-\alpha_{0}\right)+\frac{\tilde{\sigma}}{\tau}\right] \frac{\tilde{G}\left(\bar{h}_{i}\right)}{\bar{h}^{\prime}\left(\bar{h}_{i}\right)}, \quad i=1,2,3,4 .
$$


Taking $i=1$ in equation (92), and remembering that $\bar{h}_{1}=0$, gives

$$
w_{1}(\omega)=i \omega^{-1}\left(\frac{\tilde{\sigma}^{2}}{\alpha_{0} \tau}\right) \frac{\tilde{G}(0)}{\bar{h}^{\prime}(0)}=-i \omega^{-1}\left(\frac{\tilde{\sigma}^{2}}{\alpha_{0} \tau}\right) \frac{\tilde{W}_{1} \tilde{W}_{2}}{\bar{h}_{2} \bar{h}_{3} \bar{h}_{4}} .
$$

If $\bar{h}_{2}>0$ then $\operatorname{Im} w_{1}(\omega)<0$ meaning that $w_{1}(\omega)$ is stable. On the other hand, if $\tilde{p}_{1}<0$ inequalities (89) show that $\bar{h}_{2}<0$ and so now $\operatorname{Im} w_{1}(\omega)>0$ and $w_{1}(\omega)$ is unstable.

Taking $i=2$ in equation (92) gives

$$
\begin{aligned}
w_{2}(\omega) & =\bar{h}_{2}+i \omega^{-1}\left(\frac{\tilde{\sigma}}{\alpha_{0}}\right)\left[\bar{h}_{2} \varepsilon^{1 / 2} \alpha_{0}^{-1}\left(\alpha_{1}-\alpha_{0}\right)+\frac{\tilde{\sigma}}{\tau}\right] \frac{\tilde{G}\left(\bar{h}_{2}\right)}{\bar{h}^{\prime}\left(\bar{h}_{2}\right)}, \\
& =\bar{h}_{2}+i \omega^{-1}\left(\frac{\tilde{\sigma}}{\alpha_{0}}\right)\left[\bar{h}_{2} \varepsilon^{1 / 2} \tau \alpha_{0}^{-1}\left(\alpha_{1}-\alpha_{0}\right)+\frac{\tilde{\sigma}}{\tau}\right] \frac{\left(\bar{h}_{2}-\tilde{W}_{1}\right)\left(\bar{h}_{2}-\tilde{W}_{2}\right)}{\bar{h}_{2}\left(\bar{h}_{2}-\bar{h}_{3}\right)\left(\bar{h}_{2}-\bar{h}_{4}\right)} .
\end{aligned}
$$

If $\bar{h}_{2}>0$, then from equation $(94)_{2}, w_{2}(\omega)$ is unstable. If $\tilde{p}_{1}<0$, then $\bar{h}_{2}<0$ and so $w_{2}(\omega)$ is stable provided that

$$
\bar{h}_{2} \varepsilon^{1 / 2} \alpha_{0}^{-1}\left(\alpha_{1}-\alpha_{0}\right)+\tilde{\sigma} / \tau>0 .
$$

From equation (92) and inequalities (88) or (89) we see that $w_{3}(\omega)$ and $w_{4}(\omega)$ are unstable at high frequency.

Summarizing, in the high-frequency limit the branch $w_{1}(\omega)$ is stable if $\bar{h}_{2}>0$ and the branch $w_{2}(\omega)$ is stable if $\bar{h}_{2}<0$ and also equation (95) holds. In all other cases each branch $w_{i}(\omega)$ is unstable.

\subsection{Numerical results}

In Figure 6 we have taken $\tilde{p}_{1}>0$. The branch $w_{0}(\omega)$ beginning at the origin is unstable for low frequency and stable for high frequency in each part of the Figure, as is consistent with equations (45) and (46) for low frequencies and with equations (92) and (93) for high frequencies. All the other branches begin to the right of this branch. If $\alpha_{0}$ and $\alpha_{1}$ are small enough all the branches $w_{i}(\omega), i=2,3,4$, are stable at low frequency and unstable at high frequency. This can be seen in the first subfigures of Figure 6 where $\alpha_{0}$ and $\alpha_{1}$ are small. As $\alpha_{0}$ and $\alpha_{1}$ increase, all branches $w_{i}(\omega), i=2,3,4$, become unstable at low and high frequencies, see subfigure (f).

In Figure 7 we have taken $\tilde{p}_{1}<0$. The branch $w_{1}(\omega)$ beginning at $w=\tilde{p}_{1}$ is unstable at low frequency and stable at high frequency in each part of the Figure. All the other branches begin to the right of this branch and are stable at low frequency and unstable at high frequency.

In Figure 8 we illustrate the exceptional case $\tilde{p}_{1}=0$. Two branches now emanate from the origin, namely, $w_{0}(\omega)$ and $w_{1}(\omega)$, each changing its stability nature at an intermediate frequency. The branch with argument $-\pi / 4$ at zero frequency is stable at low frequency but becomes unstable at high frequency. On the other hand, the branch with argument $3 \pi / 4$ at zero frequency is unstable at low frequency but becomes stable at high frequency. The other branches behave similarly to those in Figures 6 and 7 . 


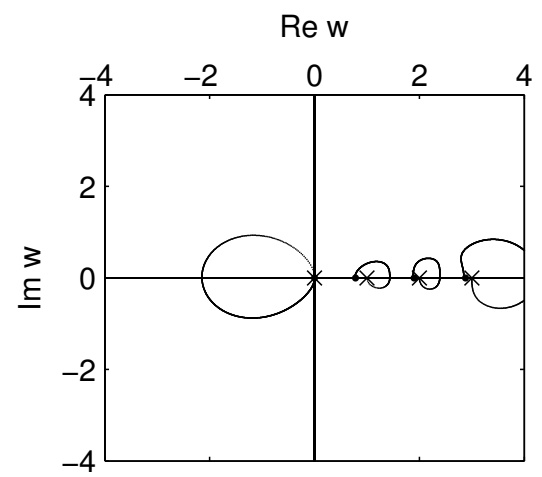

(a) $\alpha_{0}=0.02, \alpha_{1}=0.03$

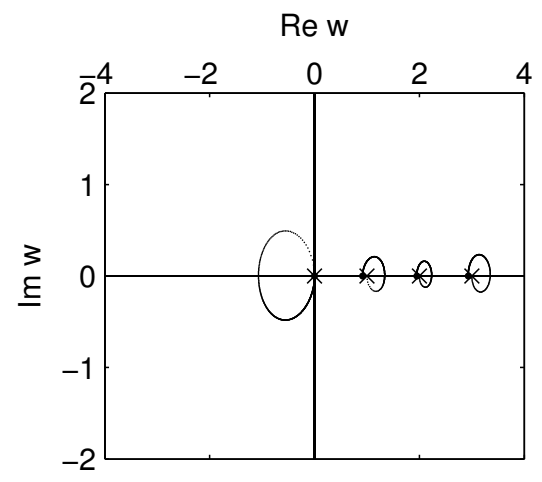

(c) $\alpha_{0}=0.25, \alpha_{1}=0.3$

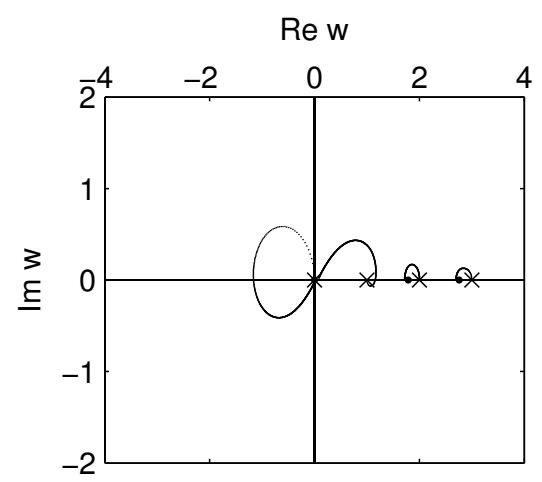

(e) $\alpha_{0}=0.4, \alpha_{1}=1$

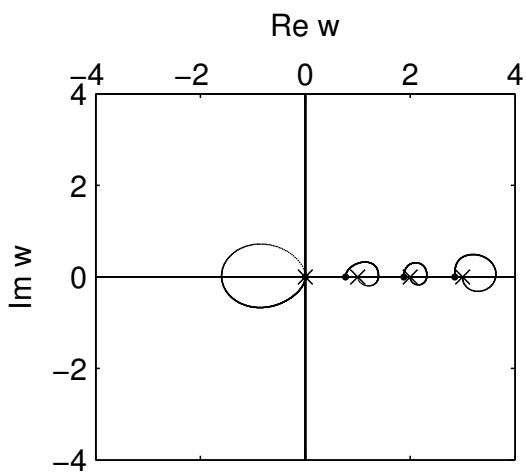

(b) $\alpha_{0}=0.1, \alpha_{1}=0.15$

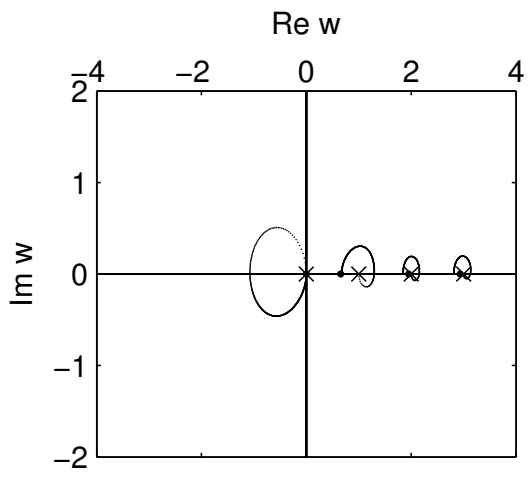

(d) $\alpha_{0}=0.3, \alpha_{1}=0.5$

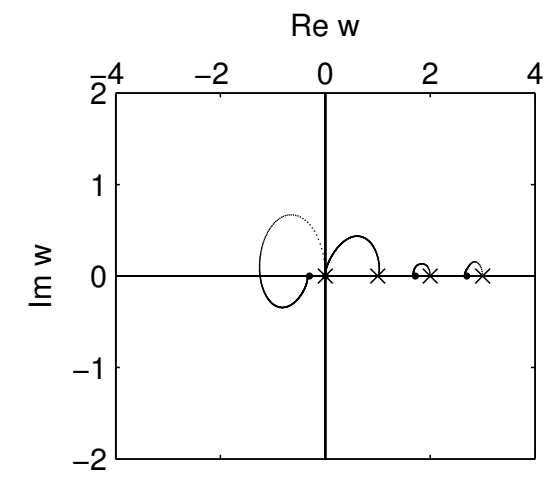

(f) $\alpha_{0}=0.5, \alpha_{1}=1.5$

Figure 6: The squared wave speeds of constrained anisotropic generalized temperature-ratedependent thermoelasticity. For each part, $\tilde{p}_{1}=1, \tilde{p}_{2}=2, \tilde{p}_{3}=3, \tilde{W}_{1}=1.5, \tilde{W}_{2}=2.5$, $\tilde{\sigma}=1, \varepsilon=1$. 

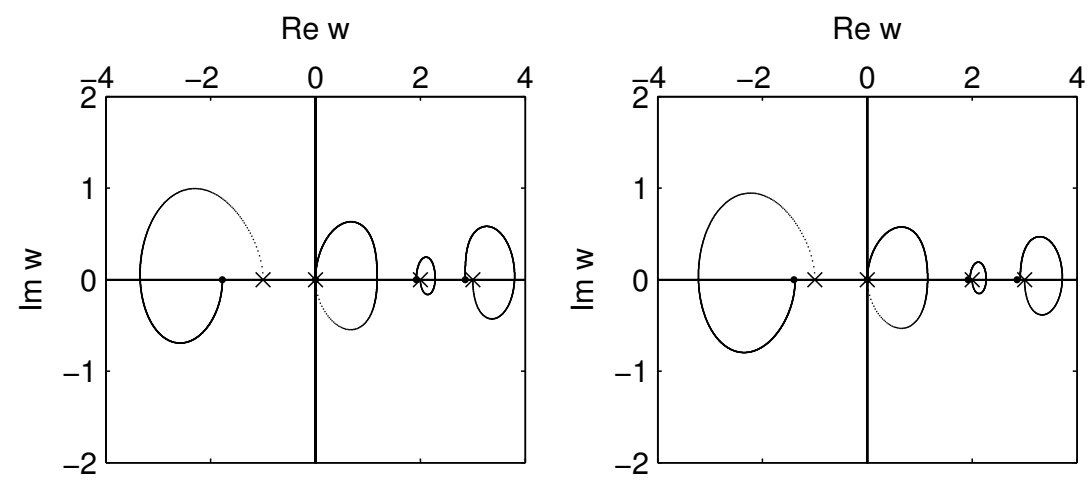

(a) $\alpha_{0}=0.01, \alpha_{1}=0.02$

(b) $\alpha_{0}=0.02, \alpha_{1}=0.03$

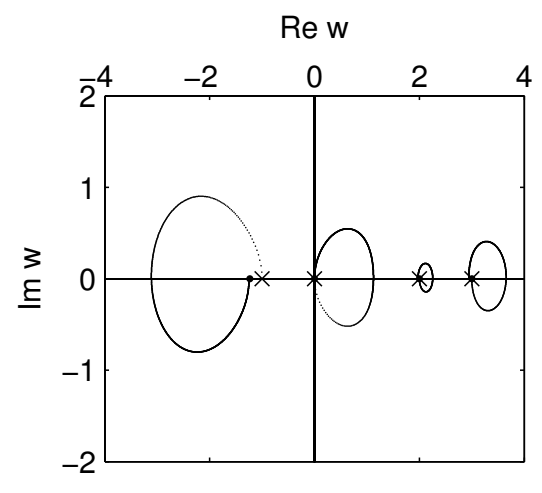

(c) $\alpha_{0}=0.03, \alpha_{1}=0.04$

Rew

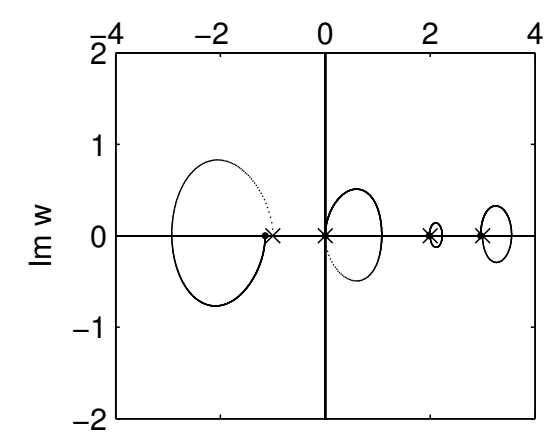

(d) $\alpha_{0}=0.05, \alpha_{1}=0.06$

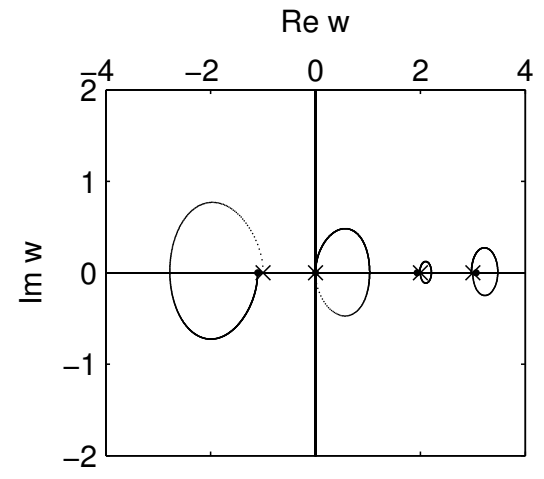

(e) $\alpha_{0}=0.07, \alpha_{1}=0.08$

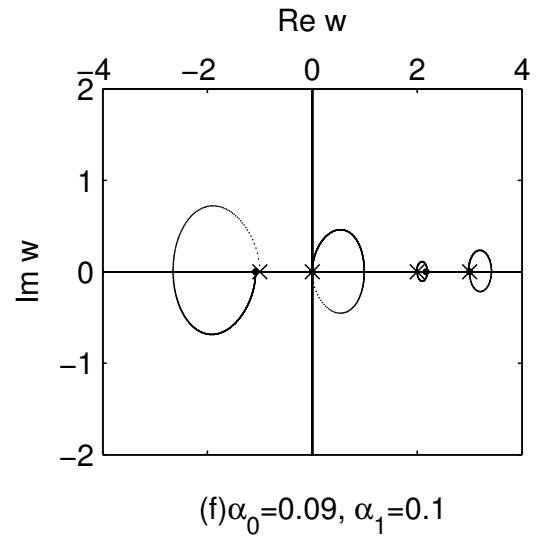

Figure 7: The squared wave speeds of constrained anisotropic generalized temperature-ratedependent thermoelasticity. For each part, $\tilde{p}_{1}=-1, \tilde{p}_{2}=2, \tilde{p}_{3}=3, \tilde{W}_{1}=1.5, \tilde{W}_{2}=2.5$, $\tilde{\sigma}=1, \varepsilon=1$. 


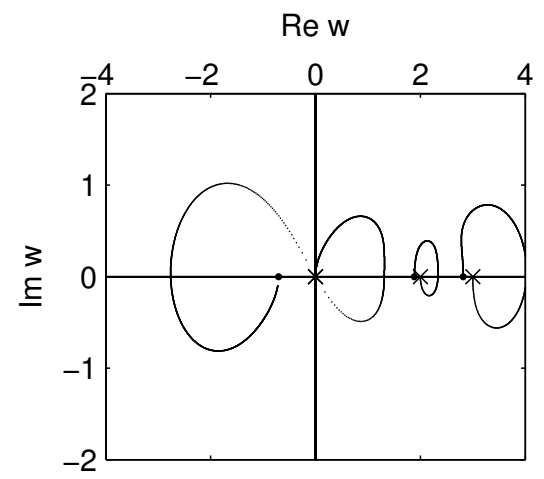

(a) $\alpha_{0}=0.01, \alpha_{1}=0.02$

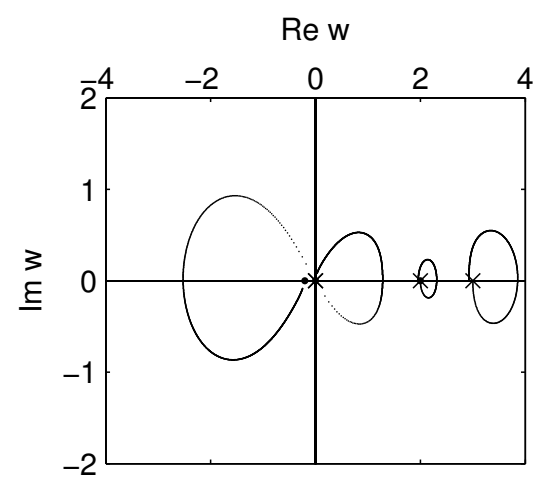

(c) $\alpha_{0}=0.03, \alpha_{1}=0.04$

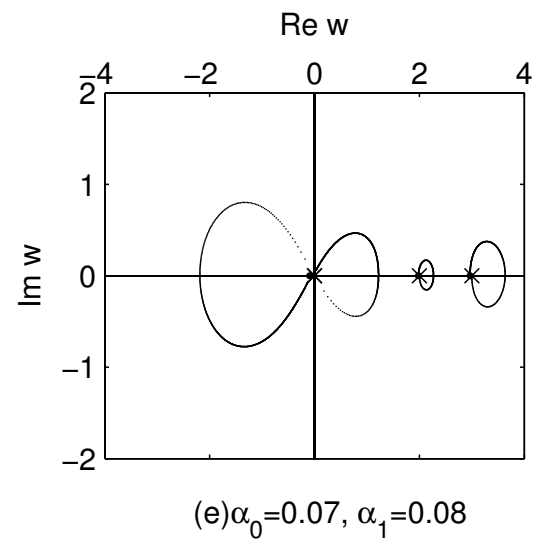

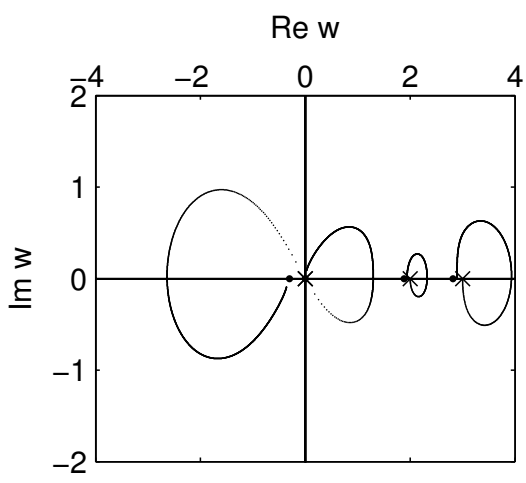

(b) $\alpha_{0}=0.02, \alpha_{1}=0.03$

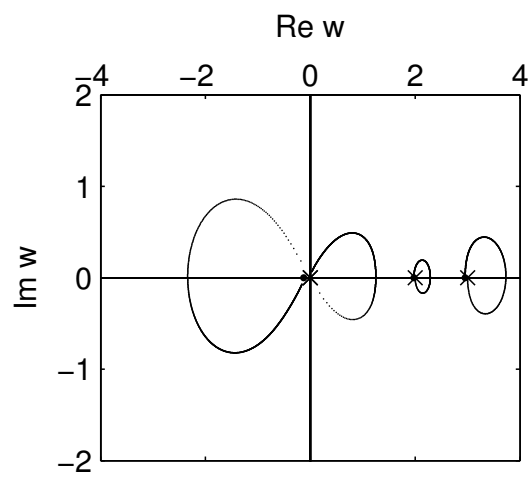

(d) $\alpha_{0}=0.05, \alpha_{1}=0.06$

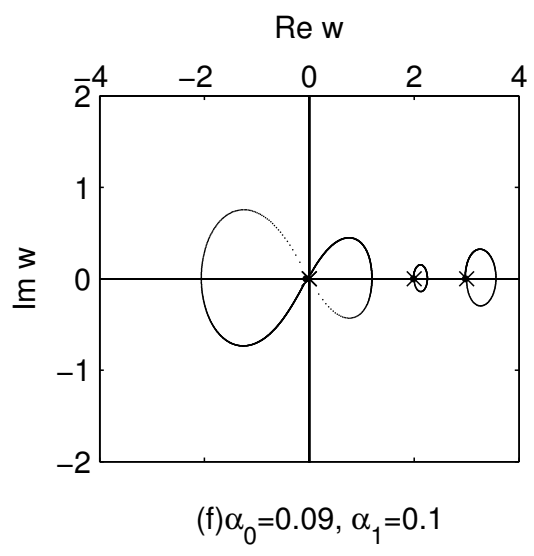

Figure 8: The squared wave speeds of constrained anisotropic generalized temperature-ratedependent thermoelasticity. For each part, $\tilde{p}_{1}=0, \tilde{p}_{2}=2, \tilde{p}_{3}=3, \tilde{W}_{1}=1.5, \tilde{W}_{2}=2.5$, $\tilde{\sigma}=1, \varepsilon=1$. 


\section{Constrained isotropic generalized temperature-rate- dependent thermoelasticity}

We specialize to the isotropic case the results of the previous section in the anisotropic case following once again the methods of Alharbi and Scott [20].

\subsection{The secular equation}

We may follow through the methods of the previous section for the isotropic case, as we did in Section 4 for the unconstrained material, or simply employ the equalities (77) in the anisotropic secular equation (84) and the definitions (40) to obtain

$$
w\left(w-\tilde{p}_{1}\right)-\frac{i \omega \tilde{\sigma}}{1-i \omega \alpha_{0}}\left[w \varepsilon^{1 / 2}\left(\alpha_{1}-\alpha_{0}\right)-\frac{\tilde{\sigma}}{1-i \omega \tau}\right]=0
$$

with $\tilde{p}_{1}$ still defined by equation (75). Putting $\tau=0$ we revert to equation (74), as expected.

Equation (96) is the secular equation for longitudinal waves in isotropic generalized temperature-rate-dependent thermoelasticity which is constrained by a deformation-temperature constraint. Of course, the two transverse waves continue to have squared wave speed given by $w=\mu$, as at equation (72).

\subsection{Low-frequency expansions}

When $\omega \rightarrow 0$ the relaxation time $\tau$ in equation (96) does not appear in the solutions so the low-frequency behaviour is exactly as in Section 4.2 . The branches $w_{0,1}(\omega)$ continue to be given by equation (78) and the stability analysis is exactly as in Section 4.2.

In the exceptional case $\tilde{p}_{1}=0$, once again the approximations (80) hold.

\subsection{High-frequency expansions}

Written in terms of $\zeta=\omega^{-1}$ equation (96) becomes

$$
w\left(w-\tilde{p}_{1}\right)+\frac{\tilde{\sigma}}{\alpha_{0}\left(1+i \zeta / \alpha_{0}\right)}\left[w \varepsilon^{1 / 2}\left(\alpha_{1}-\alpha_{0}\right)-\frac{i \zeta \tilde{\sigma}}{\tau(1+i \zeta / \tau)}\right]=0 .
$$

This equation is consistent with the anisotropic form equation (85) if we assume the equalities (77).

On taking $\zeta=0$ in equation (97) we see that the high-frequency roots are given by

$$
\bar{h}_{1}=0, \quad \bar{h}_{2}=\tilde{p}_{1}-\tilde{\sigma} \varepsilon^{1 / 2} \alpha_{0}^{-1}\left(\alpha_{1}-\alpha_{0}\right) .
$$

The difference between this and equations (88) and (89) is that here $\bar{h}_{2}$ is known explicitly. For small $\zeta$ equation (97) is well approximated by

$$
w\left(w-\bar{h}_{2}\right)-i \zeta \frac{\tilde{\sigma}}{\alpha_{0}}\left[w \varepsilon^{1 / 2} \alpha_{0}^{-1}\left(\alpha_{1}-\alpha_{0}\right)+\frac{\tilde{\sigma}}{\tau}\right]=0
$$


which is consistent with equation (91) on assuming that $\bar{h}_{3}=\bar{h}_{4}=\tilde{W}_{1}=\tilde{W}_{2}=\mu$.

The two high-frequency branches of equation (99) are well approximated by

$$
\begin{aligned}
w_{1}(\omega) & =-i \omega^{-1} \frac{\tilde{\sigma}^{2}}{\alpha_{0} \tau \bar{h}_{2}} \\
w_{2}(\omega) & =\bar{h}_{2}+i \omega^{-1} \frac{\tilde{\sigma}}{\alpha_{0} \bar{h}_{2}}\left[\bar{h}_{2} \varepsilon^{1 / 2} \alpha_{0}^{-1}\left(\alpha_{1}-\alpha_{0}\right)+\frac{\tilde{\sigma}}{\tau}\right] .
\end{aligned}
$$

The first of these is stable if $\bar{h}_{2}>0$, and unstable if $\bar{h}_{2}<0$, where $\bar{h}_{2}$ is defined by equation $(98)_{2}$. The second is unstable if $\bar{h}_{2}>0$, and stable if $\bar{h}_{2}<0$ and also

$$
\bar{h}_{2} \varepsilon^{1 / 2} \alpha_{0}^{-1}\left(\alpha_{1}-\alpha_{0}\right)+\tilde{\sigma} / \tau>0 .
$$

\subsection{Numerical results}

Figure 9 illustrates the two longitudinal waves plotted for various values of $\tilde{\sigma}$. The branch $w_{1}(\omega)$ starts from the point $w=\tilde{p}_{1}$, defined by equation $(75)_{3}$, and ends at the point $w=\bar{h}_{2}$, defined by equation $(98)_{2}$. The branch $w_{2}(\omega)$ starts and ends at the origin. At low frequency $w_{1}(\omega)$ is stable and $w_{2}(\omega)$ is unstable and the situation is reversed at high frequency. 
(a) $\tilde{\sigma}=0.3 \tilde{\sigma}_{c}$

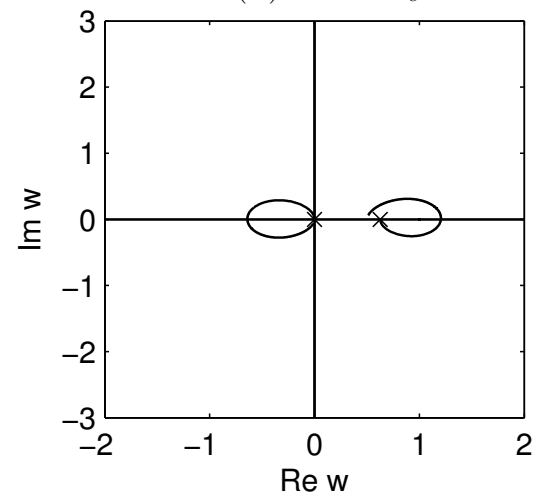

(c) $\tilde{\sigma}=\tilde{\sigma}_{c}$

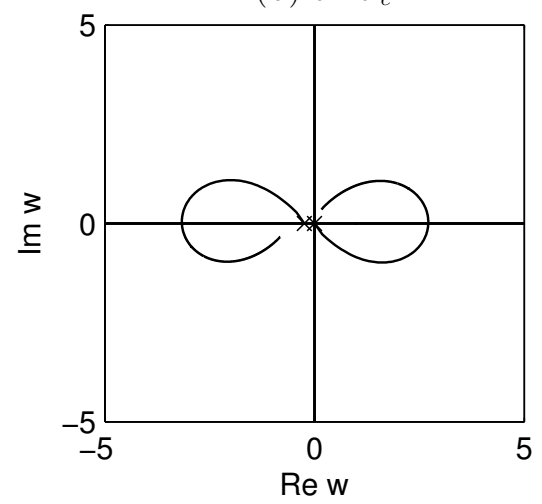

(e) $\tilde{\sigma}=5 \tilde{\sigma}_{c}$

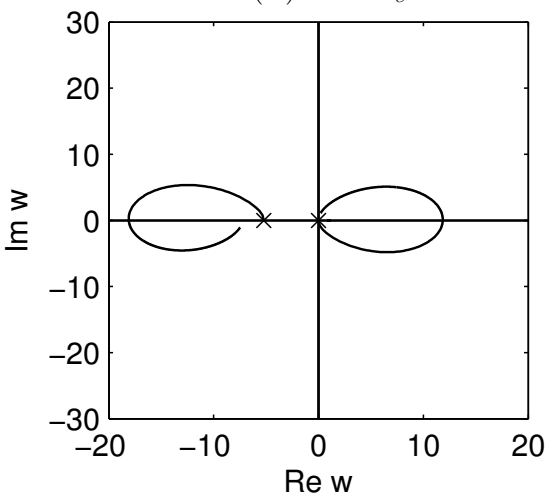

(b) $\tilde{\sigma}=0.5 \tilde{\sigma}_{c}$

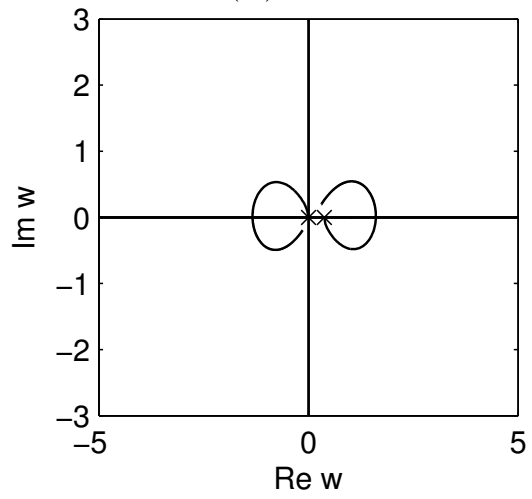

(d) $\tilde{\sigma}=3 \tilde{\sigma}_{c}$

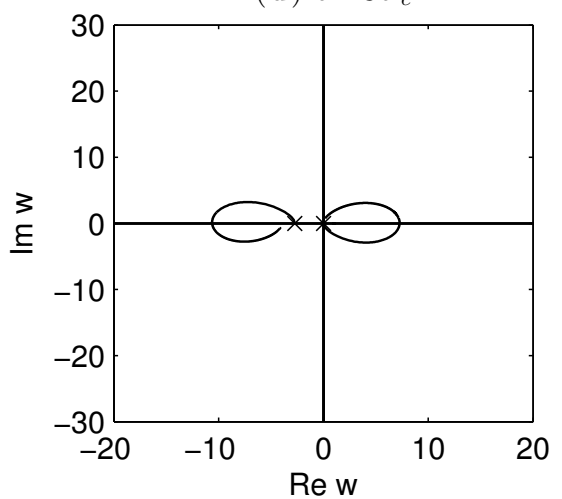

(f ) $\tilde{\sigma}=10 \tilde{\sigma}_{c}$

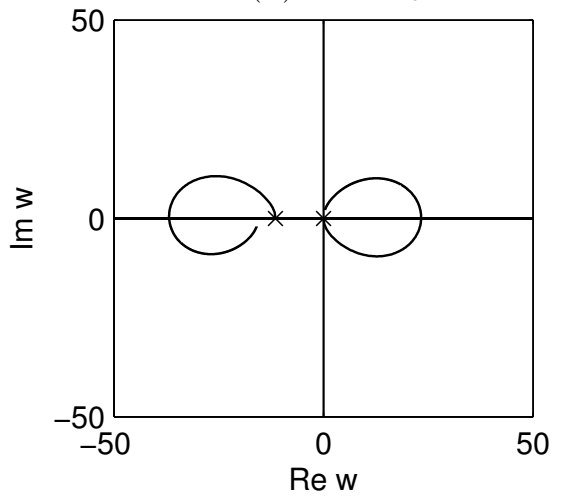

Figure 9: The longitudinal squared wave speeds of constrained isotropic generalized temperature-rate-dependent thermoelasticity. For each part, $\varepsilon=1, \alpha_{0}=0.01, \alpha_{1}=$ $0.02, \tau=0.01$. 


\section{Concluding remarks}

In the temperature-rate-dependent theory of thermoelasticity due to Green and Lindsay [13] it was shown, in the fully anisotropic case, that each of the four thermoelastic waves that may travel in each direction is stable, that is, the amplitude of an initially small disturbance remains uniformly bounded in the direction of travel for all time, see [21].

In any material we might expect an initial small disturbance either to decay to zero or remain bounded as time increases. Thus we might regard the stability of wave forms as being a possible criterion for physically reasonable response. We know that both classical and generalized thermoelasticity possess this property, see [6] and [16], respectively. We also know that temperature-rate-dependent thermoelasticity shares this stability property, see [21], and so might be an acceptable non-classical model for thermoelasticity on grounds of physically reasonable response .

However, when a deformation-temperature constraint operates in the present context of anisotropic temperature-rate-dependent thermoelasticity, we have seen in Section 3 that at least one of the four waves becomes unstable, either at low frequency or at high frequency, or at both. We saw in Section 4 that the two longitudinal waves in the constrained isotropic case exhibit the same instabilities. The same sorts of instabilities are apparent when the theory is extended to constrained generalized temperature-rate-dependent thermoelasticity, see Section 5 for the anisotropic case and Section 6 for the isotropic case. Thus in all cases at least one of the waves in constrained temperature-rate-dependent thermoelasticity or constrained generalized temperature-rate-dependent thermoelasticity is unstable.

The undesirable effects of instabilities may possibly be circumvented by assuming the constraints to hold only approximately or by using the alternative theory of deformationentropy constraints. A deformation-entropy constraint is one that connects deformation gradient with entropy, rather than temperature. Such constraints were first discussed in [22] and further developed in $[9,10,11,12]$ where it was found that the instabilities of deformation-temperature constraints are removed by considering instead deformationentropy constraints. Casey [23] considers deformation-entropy constraints from a different viewpoint and discusses the relationship between these constraints and deformationtemperature constraints. Rooney and Bechtel [24] introduce a wide variety of thermomechanical constraints. Some of them involve the entropy yet are unstable. None of these authors has considered the present case of thermomechanically constrained temperaturerate-dependent materials.

Acknowledgement A.M. Alharbi wishes to thank Taif University and the Saudi Arabian Ministry of Higher Education for their financial support during her PhD studies. The authors thank one of the referees for many insightful comments and for further references. 


\section{References}

[1] Truesdell, C and Noll, W. The non-linear field theories of mechanics, Handbuch der Physik, III/3, Springer, New York, (1965).

[2] Green, AE, Naghdi, PM and Trapp, JA . Thermodynamics of a continuum with internal constraints, International Journal of Engineering Science, 8, 891-908 (1970).

[3] Casey, J and Krishanaswamy, S. A characterization of internally constrained thermoelastic materials, Mathematics and Mechanics of Solids, 3, 71-89 (1998).

[4] Chadwick, P. Interchange of modal properties in the propagation of harmonic waves in heat-conducting materials, Bulletin of the Australian Mathematical Society, 8, 75-92 (1973).

[5] Chadwick, P. Basic properties of plane harmonic waves in a prestressed heatconducting elastic material Journal of Thermal Stresses, 2, 193-214 (1979).

[6] Scott, NH. A theorem in thermoelasticity and its application to linear stability, Proc. R. Soc. Lond. A, 424, 143-153 (1989).

[7] Chadwick, P, Whitworth, AM and Borejko, P. Basic theory of small-amplitude waves in a constrained elastic body, Arch. Ration. Mech. Analysis, 87, 339-354 (1985).

[8] Chadwick, P and Scott, NH. Linear dynamical stability in constrained thermoelasticity, I. Deformation-temperature constraints, Q. Jl Mech. Appl. Math. 45, 641-650 (1992).

[9] Scott, NH. Connections between deformation-temperature and deformation-entropy constraints and near-constraints in thermoelasticity, International Journal of Engineering Science, 34, 1689-1704 (1996).

[10] Leslie, DJ and Scott, NH. Incompressibility at uniform temperature or entropy in isotropic thermoelasticity, Q. JI Mech. Appl. Math., 51, 191-211 (1998).

[11] Leslie, DJ and Scott, NH. Wave stability for incompressibility at uniform temperature or entropy in generalized isotropic thermoelasticity. Q. JI Mech. Appl. Math., 53, 1-25 (2000).

[12] Leslie, DJ and Scott, NH. Wave stability for constrained materials in anisotropic generalized thermoelasticity, Mathematics and Mechanics of Solids, 9, 513-542 (2003).

[13] Green, AE and Lindsay, KA. Thermoelasticity, J. Elasticity, 2, 1-7 (1972).

[14] Green, AE and Laws, N. On the entropy production inequality, Arch. Rat. Mech. Anal., 45, 47-53 (1972).

[15] Lord, HW and Shulman, Y. A generalized dynamical theory of thermoelasticity, J. Mech. Phys. Solids, 15, 299-309 (1967). 
[16] Scott, NH. The stability of plane waves in generalised thermoelasticity. In Elastic Wave Propagation, Eds McCarthy, MF and Hayes, MA. 623-628. North-Holland: Amsterdam, 1989.

[17] Ignaczak, J. Linear dynamic thermoelasticity - a survey, Shock Vibration Digest, 13, 3-8 (1980).

[18] Chandrasekharaiah, DS. Thermoelasticity with second sound: A review, Applied Mechanics Reviews, 39, 355-376 (1986).

[19] Straughan, B. Heat Waves. New York: Springer, 2011.

[20] Alharbi, AM and Scott, NH. Wave stability in isotropic temperature-ratedependent thermoelasticity. Mathematics and Mechanics of Solids (2016) DOI: $10.1177 / 1081286516638778$

[21] Alharbi, AM and Scott, NH. Wave stability in anisotropic generalized temperaturerate-dependent thermoelasticity. IMA Journal of Applied Mathematics, 81, 750-778 (2016). DOI: 10.1093/imamat/hxw022

[22] Scott, NH. Linear dynamical stability in constrained thermoelasticity II. Deformationentropy constraints. Q. Jl Mech. Appl. Math. 45, 651-662 (1992).

[23] Casey, J. Strain-temperature and strain-entropy internal constraints in finite thermoelasticity. Published in Finite Thermoelasticity, AMD Vol. 236, pp. 45-65 (edited by J. Casey \& R. Abeyaratne), The American Society of Mechanical Engineers, New York, (1999).

[24] Rooney, FJ and Bechtel, SE. Constraints, constitutive limits, and instability in finite thermoelasticity. J. Elasticity, 74, 109-133 (2004). 